\author{
Aus dem Fachbereich Medizin \\ der Johann Wolfgang Goethe-Universität \\ Frankfurt am Main
}

Klinik für Anästhesiologie, Intensivmedizin und Schmerztherapie

Direktor: Prof. Dr. med. Dr. phil. Kai Zacharowski, F.R.C.A.

\title{
Stellenwert der Thrombelastometrie für das Monitoring von Faktor XIII
}

\author{
Dissertation \\ zur Erlangung des Doktorgrades der Medizin \\ des Fachbereichs Medizin \\ der Johann Wolfgang Goethe-Universität \\ Frankfurt am Main \\ vorgelegt von \\ Jan-Ove Sanders \\ aus Neustadt am Rübenberge
}

Frankfurt am Main, 2015 
Dekan: Prof. Dr. Josef M. Pfeilschifter Referent: PD. Dr. med. Christian Weber Korreferent: Prof. Dr. Axel Haferkamp Tag der mündlichen Prüfung: 14.12.2015 


\section{Inhaltsverzeichnis}

1 Einleitung 9

2 Material und Methoden 12

2.1 Studiendesign 12

2.1.1 Ethikantrag 12

2.1.2 Aufklärung und Einwilligung 12

2.1.3 Studiendesign 12

2.1.4 Patientengut 13

2.1.5 Einschlusskriterien 13

2.1.6 Ausschlusskriterien 14

2.2 Anästhesie und Patientenmonitoring 14

$\begin{array}{lll}2.3 & \text { Datenerhebung } & 16\end{array}$

2.4 Gerinnungsdiagnostik 17

2.4.1 Konventionelle Laboranalysen zur Gerinnungsdiagnostik 17

$\begin{array}{ll}2.4 .2 \text { Faktor XIII-Analyse } & 17\end{array}$

$\begin{array}{ll}2.4 .3 \text { Thrombelastometrie } & 17\end{array}$

2.5 Statistische Auswertung 27

3 Ergebnisse 28

3.1 Präoperative Kenngrößen 28

3.1.1 Beschreibung des Patientenkollektivs 28

3.1.2 Beschreibung soziodemographischer Merkmale 28

3.2 Ergebnisse der perioperativen Parameter 29

3.2.1 Thrombelastometrische Auswertung 29

3.2.2 Konventionelle Gerinnungsdiagnostik und Faktor XIII$\begin{array}{ll}\text { Einzelfaktorenanalyse } & 34\end{array}$

3.3 Multiple lineare Regressionsanalyse $\quad 40$

4 Diskussion 41

5 Zusammenfassung $\quad 49$

6 Literaturverzeichnis $\quad 51$

$\begin{array}{lll}7 & \text { Anhang } & 59\end{array}$

8 Schriftliche Erklärung $\quad 64$ 
9 Danksagung

10 Lebenslauf 


\section{$\underline{\text { Tabellenverzeichnis }}$}

Tabelle 1: $\quad$ Der Karnofsky-Score

Tabelle 2: $\quad$ Referenzbereiche der ROTEM ${ }^{\circledR}$-Parameter

Tabelle 3: $\quad$ Soziodemographische Merkmale

Tabelle 4: $\quad$ Ergebnisse der thrombelastometrischen Untersuchungen pro Messzeitpunkt

Tabelle 5: $\quad$ Gerinnungslabordiagnostik und FXIII-EinzelfaktorenAnalyse pro Messzeitpunkt

Tabelle 6: $\quad$ Multiple lineare Regressionsanalyse zur Analyse potentieller Prädiktoren für die $\mathrm{ML}_{60}$ des APTEM-Tests zu jedem Messzeitpunkt

\section{Abbildungsverzeichnis}

Abbildung 1: $\quad$ ROTEM ${ }^{\circledR}$ Vollblut Hämostasesystem

Abbildung 2: $\quad$ Prinzip der Rotationsthrombelastometrie $\left(\right.$ ROTEM $\left.^{\circledR}\right)$-Analyse

Abbildung 3: $\quad$ Darstellung der ROTEM ${ }^{\circledR}$-Parameter anhand des typischen thrombelastometrischen Graphen

Abbildung 4: $\quad$ Beispielgraphen der verschiedenen Testansätze

Abbildung 5: $\quad$ Veränderung der $\mathrm{ML}_{60}$-Werte [\%] im EXTEM

zwischen den Messzeitpunkten T1-T4 
Abbildung 6: Veränderung der $\mathrm{ML}_{60}$-Werte [\%] im APTEM

zwischen den Messzeitpunkten T1-T4

Abbildung 7: $\quad$ Streudiagramme mit Regressionskurven

Abbildung 8: Veränderung der Fibrinogen-Konzentration [mg/dl]

zwischen den Messzeitpunkten T1-T4

Abbildung 9: $\quad$ Veränderung der aPTT [s] zwischen den

Messzeitpunkten T1-T4

Abbildung 10: Veränderung des Hämatokrits [\%] zwischen

den Messzeitpunkten T1-T4

Abbildung 11: Veränderung der Thrombozyten-Konzentration [/nl] zwischen den Messzeitpunkten T1-T4

Abbildung 12: Veränderung der Faktor XIII-Konzentration [\%] zwischen den Messzeitpunkten T1-T4 41

Abbildung 13: Patienteninformation

Abbildung 14: Datenschutzerklärung

Abbildung 15: Einwilligungserklärung 


\section{Verwendete Abkürzungen}

A.

APTEM

aPTT

CFT

CT

dl

DMSO

EDTA

EKG

EK

EXTEM

$\mathrm{F}$

FXIII

FFP

FIBTEM

g

$\mathrm{h}$

$\mathrm{Hb}$

HEPES

HES

$\mathrm{Hkt}$

i.v.

IE

INR

$\mathrm{kg}$

KG

MCF

$\mathrm{mg}$

Min

$\mathrm{ml}$

MZP

n
Arteria

Aprotinin beeinflusstes Rotationsthrombelastogramm

Aktivierte Partielle Prothrombinzeit

Clot Formation Time

Clotting Time

Deziliter

Diethylsulfoxid

Ethylendiamintetraessigsäure

Elektrokadiogramm

Erythrozytenkonzentrat

extrinsisch aktiviertes Rotationsthrombelastogramm

Faktor

Blutgerinnungsfaktor XIII

Fresh Frozen Plasma

Thrombozyten-inhibiertes Rotationsthrombelastogramm

Gramm

Stunde

Hämoglobin

$\mathrm{N}$-2-Hyroxyethylpiperazin-N-2-Ethansulfonsäure

Hydroxyethylstärke

Hämatokrit

intravenös

Internationale Einheiten

International Normalized Ratio

Kilogramm

Körpergewicht

Maximum Clot Formation

Milligramm

Minuten

Milliliter

Messzeitpunkt

Anzahl der Messwerte/ Patienten 


$\begin{array}{ll}\text { OR } & \text { Odds Ratio } \\ \mathrm{p} & \text { Irrtumswahrscheinlichkeit } \\ \text { POC } & \text { Point-of-Care } \\ \text { PT } & \text { Prothrombinzeit } \\ \text { PTT } & \text { Partielle Prothrombinzeit } \\ \text { ROTEM }{ }^{\circledR} & \text { Rotationsthrombelastometrie } \\ \text { RoTEG } & \text { Rotationsthrombelastographie } \\ \text { rpm } & \text { revolutions per minute } \\ \text { S } & \text { Sekunde } \\ \text { t-Pa } & \text { tissue plasminogen factor } \\ \text { Tab } & \text { Tabelle } \\ \text { TEE } & \text { Trans-Ösophageale Echokardiographie } \\ \text { TEG } & \text { Thrombelastogramm } \\ \text { TK } & \text { Thrombozyten-Konzentrat } \\ { }^{\circ} \mathrm{C} & \text { Grad Celsius } \\ \mu l & \text { Mikroliter }\end{array}$

\section{Sonderzeichen}

(B) eingetragenes Warenzeichen

$<\quad$ kleiner

$>\quad$ größer 


\section{Einleitung}

Perioperative Blutungskomplikationen stellen, insbesondere für neurochirurgisch behandelte Patienten, eine schwerwiegende Komplikation dar und sind mit einer signifikant erhöhten Morbidität und Mortalität assoziiert [1].

Der perioperative Blutverlust ist einer der Hauptgründe für die Transfusion allogener Blutprodukte [2, 3]. Transfusionen von Erythrozytenkonzentraten (EK), Gefrorenem Frischplasma (GFP) und Thrombozytenkonzentraten (TK) sind dabei unabhängige Risikofaktoren für die Entwicklung thromboembolischer, pulmonaler, hämodynamischer oder septischer Komplikationen und selbst mit einer erhöhten perioperativen Morbidität und Mortalität assoziiert [3-10].

In der Literatur wird die Prävalenz von Blutungskomplikationen im Rahmen von intrakraniellen Eingriffen (Raten zwischen 0,8\% bis 50\%) sehr unterschiedlich beschrieben [1, 11-13].

Gleichwertige Voraussetzungen für die Bildung und Aufrechterhaltung eines stabilen Blutgerinnsels sind, neben einer effizienten primären Hämostase (Thrombozytenzahl und -funktion, Von-Willebrand-Faktor), auch die plasmatische Gerinnung (extrinsische und intrinsische Gerinnungsfaktoren) und der Schutz vor (Hyper)fibrinolyse [14].

Ebenso wie die Aufrechterhaltung der Hämostase sind auch die Ursachen für das Auftreten von Koagulopathien ein Zusammenspiel komplexer Mechanismen und oftmals multifaktorieller Genese. Häufig liegen Thrombozytenfunktionsstörungen und Störungen der plasmatischen Gerinnung parallel vor [15-19]. Mitunter kann auch ein Faktor XIII-Mangel mitverantwortlich für perioperative Blutungen sein.

Faktor XIII katalysiert die Quervernetzung zwischen Fibrinmonomeren in Polymere und stabilisiert dadurch das Gerinnsel. Gleichzeitig schützt FXIII das Gerinnsel durch die Integration antifibrinolytisch wirksamer Proteine vor vorzeitigem Abbau [20-22]. Man unterscheidet den hereditären von dem erworbenen FXIII-Mangel. Während der hereditäre FXIII-Mangel mit einer Prävalenz von $1: 1.000 .000$ bis $1: 5.000 .000$ eine äußerst seltene Erkrankung darstellt, die sich oft schon früh manifestiert (z.B. durch postpartale Nabelstumpfblutungen), kommt der erworbene FXIII-Mangel deutlich häufiger vor [20]. Die Ursachen eines Faktor XIII-Mangels sind multifaktoriell bedingt. Beschrieben wurden u.a. Synthesestörungen, Verlust- bzw. Dilutionskoagulopathien sowie erhöhter Verbrauch im Rahmen disseminierter intravasaler Gerinnung [23]. 
Ferner kann ein FXIII-Defizit mit Autoimmunerkrankungen und der Ausbildung von spezifischen FXIII-Antikörpern assoziiert sein [24]. Die Plasmakonzentration von FXIII kann durch Standardgerinnungstests, wie die Prothrombinzeit (PT) oder die Partielle Prothrombinzeit (PTT), nicht abgebildet werden und muss daher mittels Einzelfaktorenanalyse bestimmt werden [20].

Es ist bekannt, dass der prädikative Wert der konventionellen Gerinnungsanalyse für prä-, intra- und postoperative Blutungsereignisse sehr gering ist [25, 26]. In der perioperativen Gerinnungsdiagnostik gewinnen bettseitig einsetzbare sogenannte Point-of-Care-Verfahren (POC) zur Gerinnungsdiagnostik für die Steuerung von zielgerichteter Hämotherapie zunehmend an Bedeutung [25, 27]. Zwar ist es derzeit noch nicht möglich, den komplexen Vorgang der Hämostase durch ein einzelnes Gerät zu erfassen, doch lassen sich die 4 Phasen der Blutgerinnung (primäre Hämostase, Thrombingenerierung, Gerinnselbildung und -stabilisierung, Fibrinolyse) durch eine Kombination von thrombelastometrischen und aggregometrischen Verfahren abbilden [25].

Aggregometrische Verfahren ermöglichen die perioperative Bestimmung der Thrombozytenfunktion und die Differenzierung potentieller Thrombozytopathien [28, 29]. Viskoelastische POC-Verfahren ermöglichen es, den Beginn und die Dynamik der Gerinnselbildung zeitlich zu erfassen sowie die Festigkeit des Clots graphisch darzustellen. Ferner kann die Gerinnselstabilität in Abhängigkeit der Zeit erfasst und so eine eventuelle Hyperfibrinolyse detektiert werden, was bisher mit keinem anderen Gerinnungstest sicher erreicht werden kann [30, 31].

Bereits nach weniger als 10 Minuten lassen sich erste Ergebnisse ablesen, die auf mögliche Störungen der Hämostase rückschließen lassen und ein rasches therapeutisches Gegensteuern ermöglichen [32].

Besonders für Patienten, die sich einem neurochirurgischen Eingriff unterziehen, wäre eine Möglichkeit zur bettseitigen Analyse der FXIII-Konzentration von großer klinischer Relevanz, da potentiell Faktor XIII assoziierte Blutungen während und nach intrakraniellen Eingriffen auf Grund der anatomischen Verhältnisse zu lebensgefährlichen Komplikationen führen können. Ein entsprechendes Monitoring könnte bei vermutetem FXIII-Mangel dabei helfen, die Indikation zur Transfusion von Fresh Frozen Plasma (FFP) bzw. die Indikation zur Applikation von Gerinnungsfaktorenkonzentraten zu stellen.

Sowohl Fallberichte als auch in-vitro-Studien legen die Vermutung nahe, dass die 
Thrombelastometrie als Verfahren zum Monitoring der FXIII-Konzentration geeignet sein könnte [33-35]. Demnach könnte eine in-vitro diagnostizierte Aprotinin-resistente Lyse als Ausdruck nicht fibrinolytisch bedingter verminderter Gerinnselstabilität mit der FXIII-Konzentration korrelieren. Bisher existieren noch keine klinischen Untersuchungen, ob es einen Zusammenhang zwischen thrombelastometrisch nachgewiesener Aprotinin-resistenter Lyse und der FXIII-Konzentration gibt.

Es wurde folgende Nullhypothese aufgestellt:

- Die Thrombelastometrie eignet sich nicht für das perioperative Monitoring der FXIII-Konzentration. 


\section{Material und Methoden}

\subsection{Studiendesign}

\subsubsection{Ethikantrag}

Die Studie mit dem Titel „Stellenwert der Thrombelastometrie für das Monitoring von Faktor XIII" wurde am 19.03.2008 bei der örtlichen Ethikkommission vorgelegt und am 20.05.2008 mit der Prüfnummer 92/08 genehmigt.

\subsubsection{Aufklärung und Einwilligung}

Die mündliche und schriftliche Aufklärung (Abb. 13-15, im Anhang Seite 58-61) der Patienten über Ziele, Inhalt und Risiken der Untersuchungen, sowie über deren praktische Durchführung, erfolgte durch die Kollegen der Klinik und Poliklinik für Neurochirurgie des Universitätsklinikums Frankfurt. Alle Patienten nahmen freiwillig an der Studie teil und unterschrieben eine entsprechende Einwilligungserklärung (Abb. 15, im Anhang Seite 62). Die Patienten wurden darüber informiert, dass sie jederzeit und ohne Begründung ihre Teilnahme an der Studie hätten widerrufen können.

\subsubsection{Studiendesign}

Bei der vorliegenden Studie handelt es sich um eine prospektive Mono-Center-Observationsstudie.

Die vorliegende Untersuchung wurde von der Klinik für Anästhesiologie, Intensivmedizin und Schmerztherapie gemeinsam mit der Klinik und Poliklinik für Neurochirurgie im Universitätsklinikum Frankfurt durchgeführt. 


\subsubsection{Patientengut}

In die durchgeführte Studie wurden insgesamt $n=26$ Patienten eingeschlossen, die sich in der Zeit vom 30.04.2008 bis zum 16.12.2009 einer elektiv geplanten Kraniotomie zur Tumorresektion unterzogen.

\subsubsection{Einschlusskriterien}

Einschlusskriterien für die Studienteilnahme waren:

- Alter > 18 Jahren

- Karnowsky-Score $>70$

\begin{tabular}{|c|c|c|}
\hline $100 \%$ & $\mathrm{ECOG}=0$ & Keine Beschwerden, keine Zeichen der Krankheit. \\
\hline $90 \%$ & $\mathrm{ECOG}=0$ & $\begin{array}{l}\text { Fähig zu normaler Aktivität, kaum oder geringe } \\
\text { Symptome. }\end{array}$ \\
\hline $80 \%$ & $\mathrm{ECOG}=1$ & $\begin{array}{l}\text { Normale Aktivität mit Anstrengung möglich, deutliche } \\
\text { Symptome. }\end{array}$ \\
\hline $70 \%$ & $\mathrm{ECOG}=1$ & $\begin{array}{l}\text { Selbstversorgung, normale Aktivität oder Arbeit nicht } \\
\text { möglich. }\end{array}$ \\
\hline $60 \%$ & $\mathrm{ECOG}=2$ & $\begin{array}{l}\text { Einige Hilfestellung nötig, selbständig in den meisten } \\
\text { Bereichen. }\end{array}$ \\
\hline $50 \%$ & $\mathrm{ECOG}=2$ & $\begin{array}{l}\text { Hilfe und medizinische Versorgung wird oft in Anspruch } \\
\qquad \text { genommen. }\end{array}$ \\
\hline $40 \%$ & $\mathrm{ECOG}=3$ & Behindert, qualifizierte Hilfe benötigt. \\
\hline $30 \%$ & $\mathrm{ECOG}=3$ & Schwerbehindert, Hospitalisation erforderlich. \\
\hline $20 \%$ & $\mathrm{ECOG}=4$ & $\begin{array}{c}\text { Schwerkrank, intensive medizinische Maßnahmen } \\
\text { erforderlich. }\end{array}$ \\
\hline $10 \%$ & $\mathrm{ECOG}=4$ & Moribund, unaufhaltsamer körperlicher Verfall. \\
\hline $0 \%$ & $\mathrm{ECOG}=5$ & Tod. \\
\hline
\end{tabular}

Tab. 1: Der Karnofsky-Score 


\subsubsection{Ausschlusskriterien}

Ausschlusskriterien waren:

- Eine bekannte Blutgerinnungsstörung bzw. auffällige Gerinnungsanamnese, abgefragt im anästhesiologischen Aufklärungsgespräch

- Dauermedikation mit Acetylsalicylsäure und/oder Clopidogrel, welche nicht mindestens für fünf Tage präoperativ pausiert wurde

- Eine präoperativ vorliegende Abweichung von den institutionellen Normalwerten einer konventionellen Gerinnungsanalyse (Prothrombinzeit (PTZ) < 16,2 Sekunden [s]; Quick > 70\% bzw. abgeleiteter INR-Wert < 1,28; aPTT 27-37 s; Fibrinogen 150-450 mg/dl; Thrombozytenzahl 150-440/ $\mu$ l)

- Schwangerschaft

- Niereninsuffizienz (Kreatinin >0,84 mg/dl)

- bekannte Leberzirrhose

- HIV-Infektion

\subsection{Anästhesie und Patientenmonitoring}

Die durchgeführten Operationen erfolgten bei allen Patienten in Allgemeinanästhesie.

Den in die Studie eingeschlossenen Patienten wurde 30 min vor dem Eingriff eine Prämedikation von 7,5 mg Midazolam (Dormicum, Roche Pharma AG, GrenzachWyhlen, Deutschland) verabreicht. Ferner wurden den Patienten im Rahmen des perioperativen Monitorings ein fünfpoliges Elektrokardiogramm (EKG), eine Blutdruckmanschette sowie ein Pulsoximeter angelegt. Die zu überwachenden Werte wurden auf dem Monitor des Narkosegerätes (Zeus, Dräger, Lübeck, Deutschland) wiedergegeben.

Über einen peripher venösen Zugang erfolgte die Induktion einer Allgemeinanästhesie mittels 1-4 $\mu \mathrm{g} / \mathrm{kg}$ Körpergewicht Sufentanil (Sufenta ${ }^{\circledR}$, JansenCilag GmbH, Neuss, Deutschland), 5 mg/kg Körpergewicht Thiopental (Trapanal ${ }^{\circledR}$, Nycomed Deutschland $\mathrm{GmbH}$, Konstanz, Deutschland) und, nach zu voriger Sicherstellung einer komplikationslosen Maskenbeatmung, mit 0,5-0,8 mg/kg Körpergewicht Rocuronium $\left(\right.$ Esmeron $^{\circledR}$, Essex Pharma GmbH, München, 
Deutschland). Nach trachealer Intubation und Initiierung einer kontrollierten Beatmung wurde die Narkose durch eine kontinuierliche Infusion von Propofol (Propofol ${ }^{\circledR}$, Fresenius Kabi GmbH, Bad Homburg, Deutschland) und Sufentanil aufrechterhalten. Es wurde ein Blasendauerkatheter angelegt. (MSP Schneider, Modell Uniklinik Frankfurt, Horb, Deutschland).

Anschließend folgte die Kanülierung der Arteria radialis, um eine invasive Druckmessung und eine arterielle Blutgasanalyse zu ermöglichen sowie die Anlage eines vierlumigen zentralen Venenkatheters (Multi-Lumen-Katheter, Arrow International Inc., Reading, USA) in Seldinger Technik.

Musste der Patient aufgrund der intrakraniellen Lokalisation des zu entfernenden Tumors in halbsitzender Lage operiert werden, wurde direkt nach Narkoseeinleitung eine transösophageale Echokardiographie durchgeführt (TEE), um ein persistierendes Foramen ovale auszuschließen.

Zur intraoperativen Volumentherapie erhielten die Patienten nach Narkoseeinleitung und bis zur postoperativen Verlegung auf die Intensivstation eine kontinuierliche Infusion von 6-10 $\mathrm{ml} / \mathrm{kg}$ Körpergewicht einer kristalloiden Infusionslösung (Sterofundin $^{\circledR}$, B. Braun Melsungen AG, Deutschland). Um einer kardiozirkulatorischen Dekompensation im Rahmen der Aufrichtung in die halbsitzende Position vorzubeugen, erhielten die Patienten nach klinikinternen Qualitätsstandards noch vor Beginn der Lagerungsmaßnahmen zusätzlich $15 \mathrm{ml} / \mathrm{kg}$ Körpergewicht kolloidale Infusionslösung $\left(6 \%\right.$ HES 130/0,4, Voluven ${ }^{\circledR} 6 \%$, Fresenius Kabi GmbH).

Erythrozytenkonzentrate wurden auf der Grundlage von klinischen Tranfusionstriggern transfundiert. Eine Indikation für hämostatische Therapie (Fresh Frozen Plasma, Thrombozytenkonzentrate, Gerinnungsfaktorkonzentrate) wurde bei diffuser Blutungsneigung auf Basis des klinikinternen Algorithmus gestellt.

Der intraoperative Blutverlust wurde aus der Differenz zwischen Saugervolumen und verwendeter Spülflüssigkeit berechnet.

Postoperativ wurden die Patienten auf die neurochirurgische Intensivstation verlegt und dort nach klinikinternem Standard behandelt.

Abhängig vom klinischen Zustand und postoperativen Verlauf erfolgte am Folgetag eine Verlegung der Patienten auf eine der chirurgisch geführten Normalstationen. 


\subsection{Datenerhebung}

Für die durchgeführten hämatologischen Analysen wurde an insgesamt vier Messzeitpunkten (T1-T4) arterielles Blut entnommen. Die ersten $2 \mathrm{ml}$ Blut wurden dabei stets verworfen.

T1 (Baseline-Messung):

Nach Einleitung der Allgemeinanästhesie und Anlage der arteriellen Kanüle

T2:

Nach Beendigung der Lagerungsmaßnahmen und vor Hautschnitt (bei Patienten, die in halbsitzender Lagerung operiert wurden, nach Gabe von $15 \mathrm{ml} / \mathrm{kg}$ Körpergewicht kolloidaler Infusionslösung)

T3:

Postoperativ bei Verlegung auf die Intensivstation

T4:

24 Stunden nach Aufnahme auf die Intensivstation

Insgesamt wurden zu jedem Messzeitpunkt (MZP) drei mit Natriumzitrat antikoagulierte $3 \mathrm{ml}$ Röhrchen (Sarstedt AG, Nürnberg, Germany) entnommen: Jeweils eines für die konventionelle Gerinnungsdiagnostik, die FXIIIEinzelfaktorenanalyse und die ROTEM ${ }^{\circledR}$-Diagnostik. Zusätzlich wurde ein $4,7 \mathrm{ml}$ EDTA-Röhrchen (Sarstedt AG) zur Bestimmung eines kleinen Blutbildes entnommen.

Die konventionelle Gerinnungsdiagnostik und die ROTEM ${ }^{\circledR}$-Analysen wurden unmittelbar nach der jeweiligen Blutentnahme durchgeführt.

Aus methodischen und ökonomischen Gründen wurde die FXIII-EinzelfaktorenAnalyse nach Abschluss der Datenerhebung als Sammelanalyse durchgeführt.

Hierzu wurde die mit Natriumzitrat antikoagulierte Blutprobe für etwa zehn Minuten zentrifugiert $\left(4{ }^{\circ} \mathrm{C}, 3 \mathrm{rpm}\right.$, Centrifuge $5702 \mathrm{R}$, Eppendorf AG, Hamburg, Deutschland), das Plasma pipettiert und bis zum Analysetag in 1,5 ml Eppendorfcups (Eppendorf AG) bei $-70^{\circ} \mathrm{C}$ eingefroren (HFU 586 basic, Thermo-Electron $\mathrm{GmbH}$, Langenselbold, Deutschland). 


\subsection{Gerinnungsdiagnostik}

\subsubsection{Konventionelle Laboranalysen zur Gerinnungsdiagnostik}

Die Thrombozytenzahl, der Hämatokrit, die Prothrombinzeit, die INR, die FibrinogenKonzentration und die aktivierte partielle Thromboplastinzeit (aPTT) wurden an einem Hämatologiesystem (Sysmex XE 2001, Sysmex GmbH, Norderstedt, Deutschland) bzw. an einem Gerinnungsanalysator (STA-R Evolution ${ }^{\circledR}$, Roche AG, Grenzach, Deutschland) im Zentrallabor des Universitätsklinikums Frankfurt bestimmt.

\subsubsection{Faktor XIII-Analyse}

Die für eine Sammelanalyse tiefgefrorenen Blutproben wurden am Analysetag zunächst in einem Wärmebad $\left(37^{\circ} \mathrm{C}\right)$ aufgetaut. Anschließend erfolgte die Bestimmung der Plasmakonzentration des Faktor XIII photometrisch durch das Behring Coagulation System $\left(\right.$ BCS $^{\circledR}$, Berichrom ${ }^{\circledR}$-Faktor XIII-Test, Siemens Healthcare Diagnostics $\mathrm{GmbH}$, Deutschland).

\subsubsection{Thrombelastometrie}

Die klassische Thrombelastographie (TEG) wurde erstmals 1948 von Hellmut Hartert aus Heidelberg beschrieben [36]. Nach einer breiten Anwendung in den 50er- und 60 er Jahren, rückte es in den folgenden Jahren insbesondere wegen einer Vibrationsempfindlichkeit und damit verbundener Messungenauigkeit in den Hintergrund und geriet dabei fast in Vergessenheit. Ein Wiederaufleben erfuhr das TEG in den letzten Jahren durch die Weiterentwicklung zur nun nicht mehr vibrationsempfindlichen Rotationsthrombelastographie (RoTEG). Aus patentrechtlichen Gründen wurde aus „Rotationsthrombelastographie“ (ROTEG) „Rotationsthrombelastometrie“ $\left(\right.$ ROTEM $\left.^{\circledR}\right)$ [37].

Mit dem ROTEM ${ }^{\circledR}$ lässt sich ein breites Spektrum der Blutgerinnung erfassen. Es können der Beginn der Gerinnselbildung, Fibrinbildung, die Polymerisierung der Fibrinmonomere, die Interaktion mit Thrombozyten, die Stabilisierung und Retraktion 
des Gerinnsels, sowie dessen Lyse graphisch dargestellt werden. Bereits etwa 10 Minuten nach Beendigung der Pipettierschritte lassen sich erste Ergebnisse ablesen, die auf mögliche Störungen der Hämostase rückschließen lassen und ein rasches therapeutisches Gegensteuern ermöglichen [30, 32].

\subsubsection{System, Zubehör und Messverfahren}

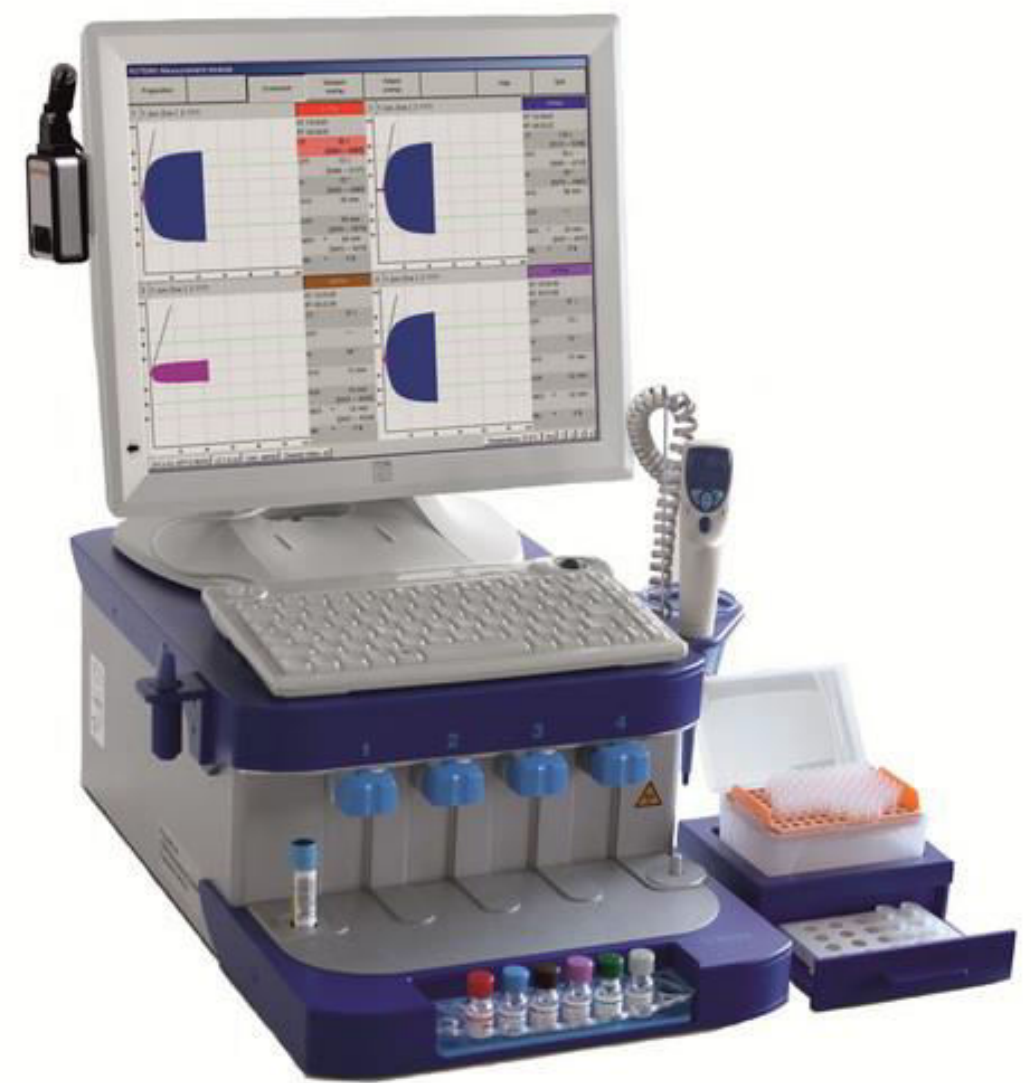

Abb. 1: ROTEM ${ }^{(\circledast)}$ Vollblut Hämostasesystem [38]

Das ROTEM ${ }^{\circledR}$ Vollblut Hämostasesystem (Pentapharm GmbH, München) setzt sich aus den folgenden Komponenten zusammen:

- Einem Messgerät, welches mit einem Computersystem verbunden ist, das die Kommunikation mit dem Notebook, die Auswertung des Signals sowie die Heizung des Wärmeblocks steuert

- Einem Notebook, das mit der aufgespielten Software das Messgerät steuert und die Daten speichert 
- Einer elektronischen Pipette, die mit dem Messgerät verbunden ist

- Optional einem Drucker

Das ROTEM ${ }^{\circledR}$ besitzt vier Messkanäle für parallele Messungen. Dabei werden die errechneten Daten fortlaufend während der Messung ausgewertet und auf dem Bildschirm des Notebooks als Diagramme und Messwerte angezeigt. Alle Ergebnisse werden auf der Festplatte des Notebooks gespeichert und können zu einem beliebigen Zeitpunkt erneut aufgerufen und bei Bedarf über den optional angeschlossenen Drucker ausgedruckt werden. Ferner ist es möglich, die Daten auf einen externen Datenträger zu übertragen und auf einem anderen Computer zu bearbeiten.

Die elektrische Pipette erlaubt eine exakte Dosierung der für Gerinnungsaktivierung notwendigen Reagenzien.

Das Messgerät enthält einen Wärmeblock, der durch seine Verbindung mit dem Küvettenhalter die Blutproben bei konstant $37^{\circ} \mathrm{C}$ hält.

Für jede Probe wird eine eigene Küvette (Cup), welche das Blut und die Reagenzien enthält, sowie ein Stempel (Pin) verwendet. Sowohl Cup als auch Pin haben eine zylindrische Form und bestehen aus Kunststoff. Das Cup wird vor der eigentlichen Messung im Küvettenhalter lokalisiert und mittels MC-Rod (measuring-cell) fixiert, der Pin auf das untere Ende der Achse des jeweiligen Kanals aufgesteckt. Die das Citratblutgemisch enthaltende Küvette (Cup) wird in dem Küvettenhalter fixiert und auf den entsprechenden Messkanal aufgeschoben. Dabei taucht der am Messkanal befestigte Stempel (Pin) in die Blutprobe ein, so dass sich ein ca. $1 \mathrm{~mm}$ breiter, mit Blut gefüllter Spalt zwischen Pin und Cup bildet. Der zylindrisch aufgebaute Pin ist dabei auf einer von einem Kugellager geführten Achse montiert und rotiert, durch Federkraft angetrieben, in einem Winkel von $4,75^{\circ}$ abwechselnd in die jeweils entgegengesetzte Richtung. Durch einen sich am oberen Ende der rotierenden Achse befindlichen Spiegel wird ein von einer Dioden-Lichtquelle erzeugter Lichtstrahl reflektiert und die entsprechende Auslenkung anhand eines CCD-Zeilensensors (charge coupled device) bestimmt. Mit einsetzender Gerinnselbildung steigt die Viskosität der Blutprobe. Die Bewegung des Pins wird in der Folge durch das sich zwischen Stempel und Küvette bildende Gerinnsel eingeschränkt. Diese Stempelrotation wird in Echtzeit in eine Amplitude umgerechnet und auf dem angeschlossenen Notebook-Monitor als ROTEM $^{\circledR}$-Kurve (TEMogramm) angezeigt. 
Die ROTEM ${ }^{\circledR}$-Kurve wird als Graph gegen die Zeit dargestellt. Die Messergebnisse werden als Zahlen auf dem Bildschirm angezeigt. Aus historischen Gründen ist der Graph doppelseitig.

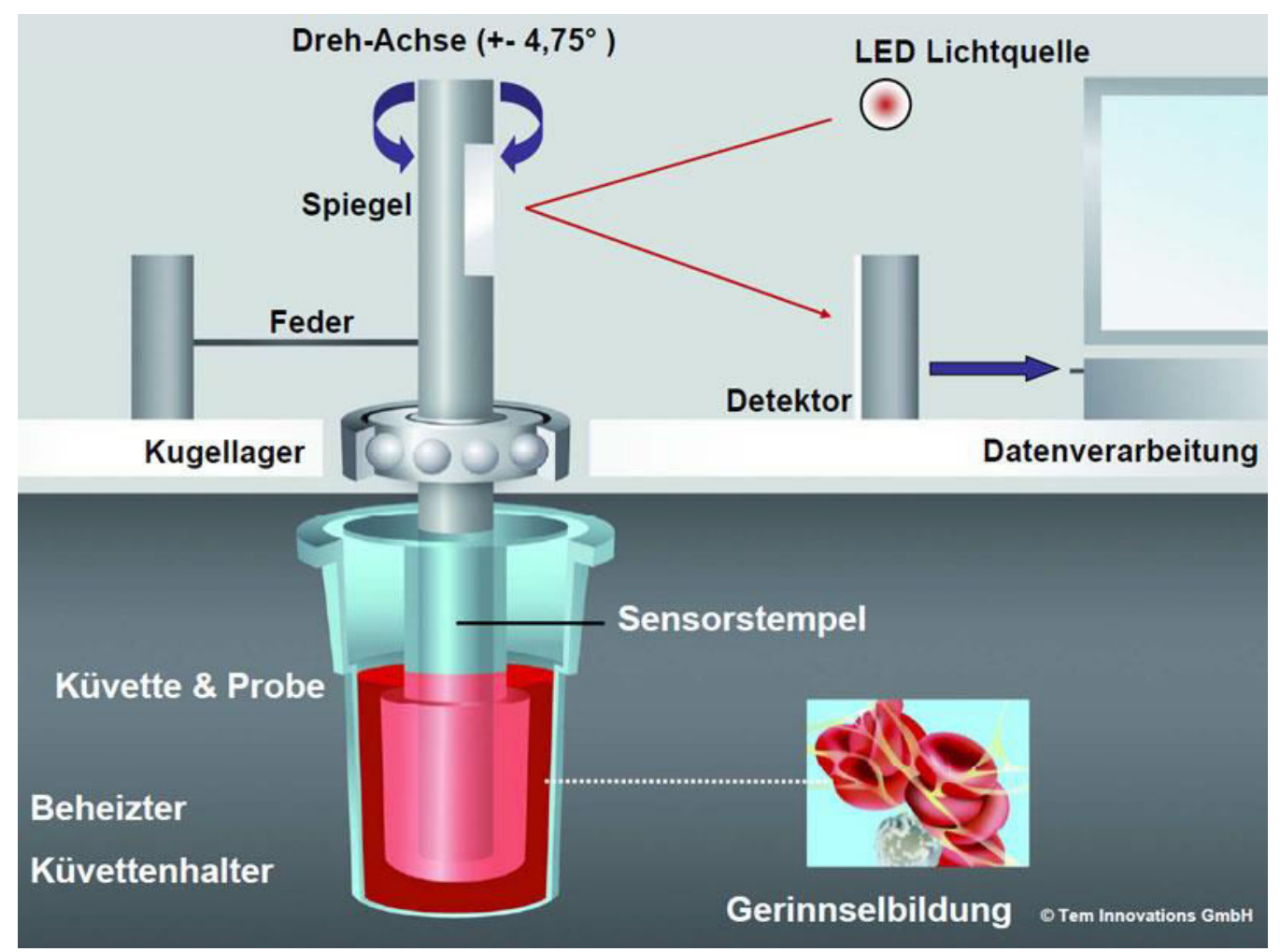

Abb. 2: Prinzip der Rotationsthrombelastometrie $\left(\mathrm{ROTEM}^{\circledR}\right)$-Analyse [38]. 


\subsubsection{Mischverfahren}

Es wurden zwei verschiedene Mischverfahren verwendet. Die elektronische Pipette ist dabei so programmiert, dass sie je nach ausgewähltem Messkanal die genaue Menge Citratblut, Reagenz und, wenn vorgesehen, Luft aufnimmt.

\section{EXTEM-Test:}

1. Pipettenspitze aufnehmen

2. Star-tem ${ }^{\circledR}$-Reagenz aufziehen, ein definiertes Volumen Luft aufnehmen, ex-tem ${ }^{\circledR}$-Reagenz aufziehen (Die beiden Reagenzien werden durch das Luftpolster in der Pipettenspitze getrennt).

3. Entleerung in die entsprechende Küvette

4. Pipettenspitzenwechsel

5. Blutprobe aufnehmen und in die Küvette entleeren. Mit derselben Pipettenspitze das Blutgemisch aufziehen und erneut in die Küvette entleeren, um ein Vermischen des Inhalts zu erreichen

6. Mit Küvette bestückten Küvettenhalter in den entsprechenden Messkanal schieben

\section{FIBTEM- und APTEM-Test}

1. Pipettenspitze aufnehmen

2. Ex-tem ${ }^{\circledR}$-Reagenz aufziehen und in die entsprechende Küvette geben

3. Pipettenspitzenwechsel

4. Fib-tem ${ }^{\circledR}$ - bzw. ap-tem ${ }^{\circledR}$-Reagenz aufnehmen und in dieselbe Küvette geben

5. Pipettenspitzenwechsel

6. Blutprobe aufnehmen und in die Küvette entleeren. Mit derselben Pipettenspitze das Blutgemisch aufnehmen und erneut in die Küvette entleeren.

7. Mit Küvette bestückten Küvettenhalter in den entsprechenden Messkanal schieben 


\subsubsection{Testparameter}

Für die durchgeführte Studie wurden folgende Testparameter ausgewertet:

CT [s] (clotting time - Gerinnungszeit)

Die CT beschreibt die Zeitspanne vom Beginn der Signalaufzeichnung bis zum Nachweis einer signifikanten Gerinnselbildung. Diese ist per Definition erreicht, sobald eine Amplitude von $2 \mathrm{~mm}$ gemessen werden kann. Sie wird in Sekunden angegeben.

CFT [s] (clot formation time - Gerinnselbildungszeit)

Die CFT beschreibt die Zeitspanne vom Ende der Gerinnungszeit (CT) bis zum Erreichen einer bestimmten Gerinnselfestigkeit, die durch das Erreichen einer Amplitude von $20 \mathrm{~mm}$ definiert wird. Sie wird in Sekunden angegeben.

MCF [mm] (maximum clot firmness - maximale Gerinnselfestigkeit)

Die MCF beschreibt die maximale Festigkeit, welche das Gerinnsel während der Messung erreicht hat. Sie wird in $\mathrm{mm}$ angegeben.

$\mathrm{ML}_{60}[\%]$ (Maximale Lyse nach 60 Minuten)

Die $\mathrm{ML}_{60}$ ist definiert als der prozentuale Anteil der Amplitude, gemessen 60 Minuten nach Beginn der Gerinnselbildung, bezogen auf die maximale Gerinnselfestigkeit (MCF). Sie wird in \% angegeben.

Im EXTEM- und APTEM-Test wurden die CT [s], die MCF [mm] und die $\mathrm{ML}_{60}$ bestimmt. Im FIBTEM-Test wurde lediglich die MCF [s] bestimmt. 


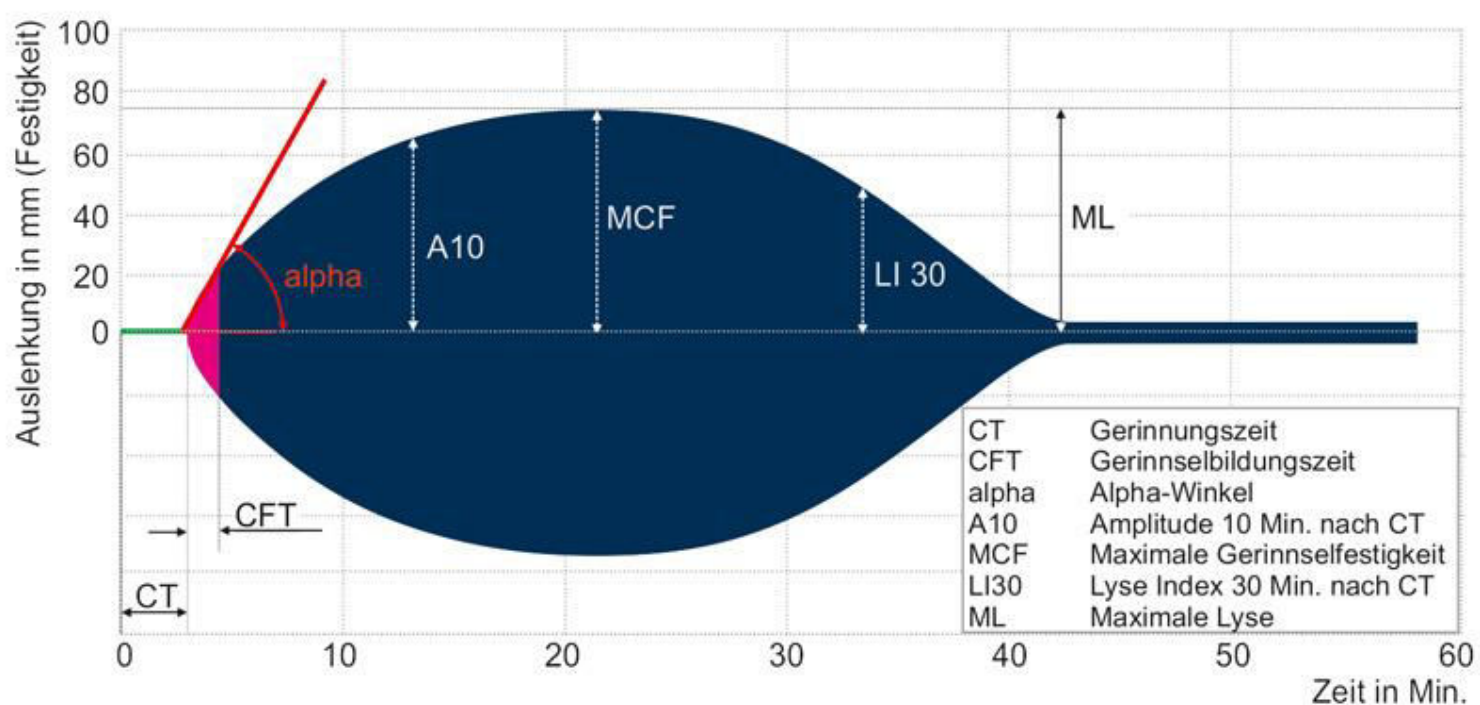

Abb. 3: Darstellung der ROTEM ${ }^{\circledR}$-Parameter anhand des typischen thrombelastometrischen Graphen [38].

\begin{tabular}{|c|c|c|c|}
\hline & EXTEM & FIBTEM & APTEM \\
\hline CT [s] & $40-80$ & & $40-80$ \\
\hline CFT [s] & $35-160$ & & $35-160$ \\
\hline MCF [s] & $50-72$ & $8-25$ & $50-72$ \\
\hline ML $60_{6}[\%]$ & $<15$ & & $<15$ \\
\hline
\end{tabular}

Tab. 2: Referenzbereiche der untersuchten ROTEM ${ }^{\circledR}$-Parameter [38]. 


\subsubsection{Reagenzien}

star-tem $^{\circledR}$ :

Besteht aus 0,2 mol/l $\mathrm{CaCL}_{2}$ in HEPES Puffer $\mathrm{pH} \mathrm{7,4}$ und 0,1\% Natriumazid.

Star-tem ${ }^{\circledR}$ dient zur Rekalzifizierung der Citratblutprobe. Je nach Untersuchung wird es zusätzlich zu Thromboplastinen (EXTEM) und partiellen Thromboplastinen in das Citratblut pipettiert und setzt den Gerinnungsprozess durch Rekalzifizierung in Gang.

\section{ex-tem ${ }^{\circledR}$ :}

Besteht aus Thromboplastin, Heparin-Inhibitor, Puffer und Konservierungsmittel. Ex-tem $^{\circledR}$ führt zu einer Aktivierung des extrinsischen Gerinnungssystems über Faktor VII.

\section{fib-tem ${ }^{\circledR}$ :}

Besteht aus Cytochalasin D / DMSO Lösung, 0,2 mol// $\mathrm{CaCl}_{2}$ in $\mathrm{HEPES}$ Puffer pH 7,4 und Konservierungsmittel. Fib-tem ${ }^{\circledR}$ wird stets zusammen mit ex-tem ${ }^{\circledR}$ verwendet und bewirkt eine Hemmung der Thrombozytenaktivität durch Cytochalasin D, so dass ein reines Fibringerinnsel entsteht.

\section{ap-tem ${ }^{\circledR}$ :}

Besteht aus Aprotinin, 0,2 mol/l CaCl2 in HEPES Puffersubstanz $\mathrm{pH} \mathrm{7,4}$ und $0,1 \%$ Natriumazid (NaN3). Ap-tem ${ }^{\circledR}$ wird stets zusammen mit Ex-tem ${ }^{\circledR}$ verwendet und bewirkt durch den enthaltenen Plasmin-Inhibitor Aprotinin, dass das im Citratblut enthaltende Plasmin sofort inaktiviert wird. 


\subsubsection{Verwendete Tests ( EXTEM, FIBTEM, APTEM)}

Für die thrombelastometrischen Testansätze wurden ausschließlich standardisierte ROTEM ${ }^{\circledR}$-Reagenzien der Firma Pentapharm (München, Deutschland) verwendet. Die präanalytische Präparation der Reagenzien erfolgte anhand des vom Hersteller vorgegebenen Protokolls.

\section{EXTEM-Test:}

Es werden mit Hilfe der Pipette $20 \mu$ star-tem $^{\circledR}$ und $20 \mu$ l ex-tem ${ }^{\circledast}$ mit $300 \mu$ l Citratblut vermischt. Durch die Zuführung von Calcium und Tissue Factor wird die Gerinnung aktiviert. Untersucht wird bei diesem Test die extrinsische Gerinnung: Faktor VII, Faktor X, Faktor V, Faktor II, Faktor I, Thrombozyten und die Fibrinolyse.

\section{FIBTEM-Test:}

Es werden mit Hilfe der Pipette $20 \mu \mathrm{l}$ ex-tem ${ }^{\circledR}$ und $20 \mu$ fib-tem ${ }^{\circledR}$ mit $300 \mu \mathrm{l}$ Citratblut vermischt. Da fib-tem ${ }^{\circledR}$ bereits Calcium enthält, ist eine zusätzliche Gabe von star-tem $^{\circledR}$ nicht nötig. Durch die Zuführung von Cytochalasin D wird der thrombozytäre Effekt auf die Gerinnselstabilität blockiert. Das entstehende Gerinnsel besteht lediglich aus gebildetem und polymerisiertem Fibrin. Der FIBTEM-Test reflektiert also die plasmatischen Bestandteile der Gerinnselstabilität, hauptsächlich die Fibrinogen/ Fibrin-Komponente.

\section{APTEM-Test:}

Es werden mit Hilfe der Pipette $20 \mu \mathrm{l} \mathrm{ex-tem}{ }^{\circledR}$ und $20 \mu \mathrm{l}$ ap-tem ${ }^{\circledR}$ mit $300 \mathrm{ml}$ Citratblut vermischt. Auch beim APTEM-Test kann auf die Zugabe von star-tem ${ }^{\circledR}$ verzichtet werden. Durch die Zuführung des Antifibrinolytikums Aprotinin wird das im Citratblut enthaltene Plasmin direkt blockiert. Untersucht wird bei diesem Test, ob eine potentielle (Hyper)fibrinolyse durch die in-vitro-Zugabe von Aprotinin hemmbar ist. 

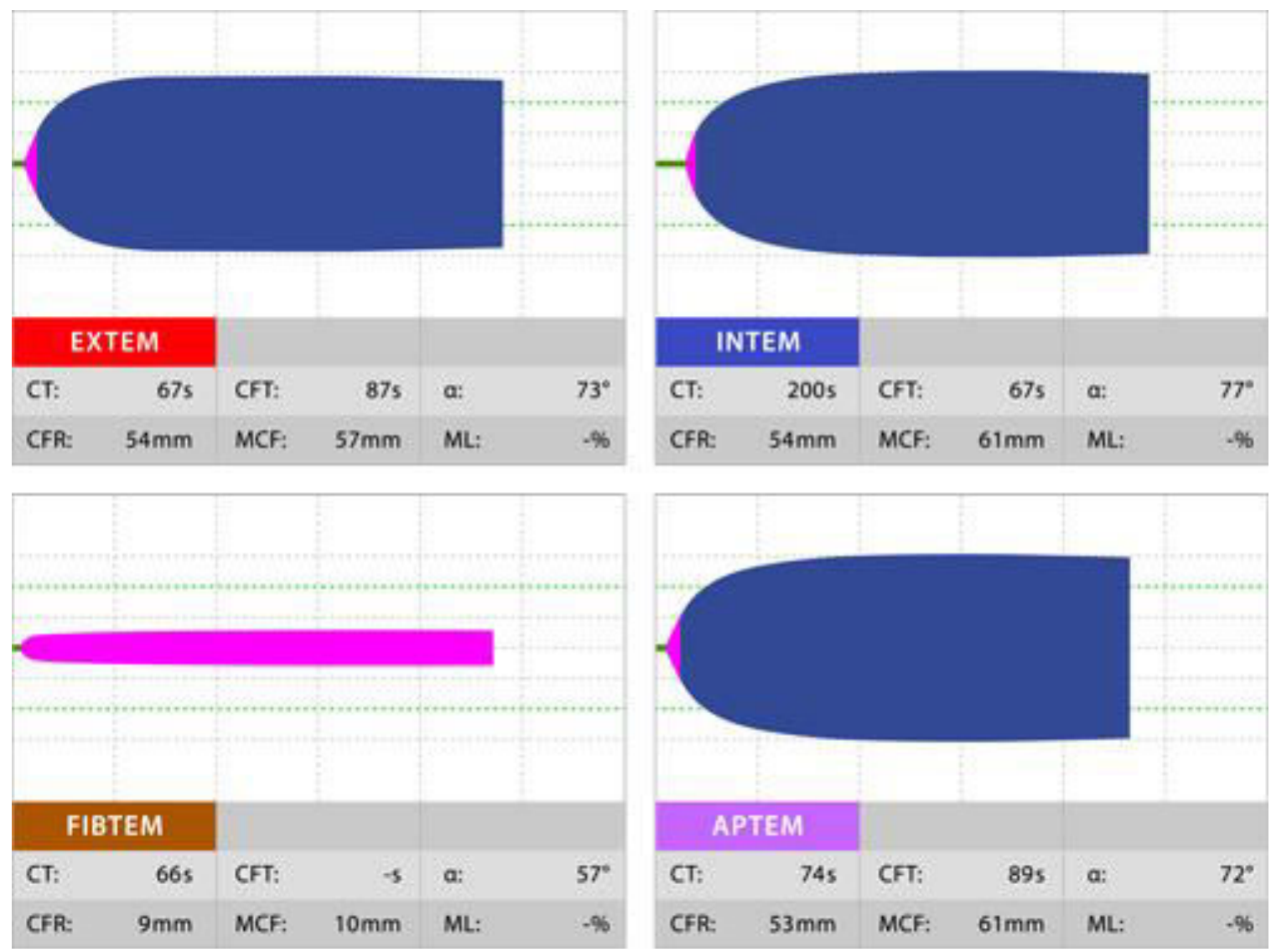

Abb. 4: Beispielgraphen der verschiedenen Testansätze [38]. 


\subsection{Statistische Auswertung}

Die statistische Auswertung sowie die graphische Darstellung der Ergebnisse wurden mit den Programmen SigmaStat 3.5 und SigmaPlot 11 (Systat Software $\mathrm{GmbH}$, Erkrath, Deutschland) durchgeführt. Bisher lagen keinerlei publizierte Daten über potentielle perioperative Veränderungen der maximalen Lyse im APTEM-Test des ROTEM $^{\circledR}$ vor. Aus diesem Grund basierte die Fallzahlanalyse auf den antizipierten Veränderungen der FXIII-Konzentration: Um bei einer gewünschten Teststärke von 0,8 eine Reduktion des FXIII gegenüber der Ausgangslage zu T1 um mindestens $10 \%$ bei einer Standartabweichung von $15 \%$ als signifikant nachweisen zu können, war eine Gruppengröße von mindestens 20 Patienten notwendig.

In Abhängigkeit von der Datenverteilung (Kolmogorov-Smirnov-Test) wurden Veränderungen gegenüber der Ausgangslage an T1 entweder mit dem gepaarten T-Test oder dem Wilcoxon-Rangsummentest errechnet. Es wurden Rangsummenkorrelationen zwischen der maximalen Lyse im APTEM-Test des ROTEM $^{\circledR}$ und der FXIII-Konzentration zu jedem der vier Messzeitpunkte errechnet. Um potentielle Prädikationen für die $\mathrm{ML}_{60}$ des APTEM-Tests zu errechnen, wurden multiple lineare Regressionsanalysen durchgeführt und dabei die Thrombozytenzahl sowie die Fibrinogen- und FXIII-Konzentration als unabhängige Variablen definiert. Die Sensitivität und Spezifität der maximalen Lyse nach 60 Minuten im APTEM-Test des ROTEM ${ }^{\circledast}$ zur Detektion eines FXIII-Defizites wurden für die Messzeitpunkte T2, T3 und T4 nach den folgenden Formeln errechnet:

- richtig positiv / (richtig positiv + falsch negativ)

- richtig negativ / (richtig negativ + falsch positiv)

Hierbei wurden die $\mathrm{ML}_{60} \leq 12 \%$ und eine FXIII-Konzentration von $\geq 70 \%$ als untere Normgrenze definiert.

Abhängig von ihrer Verteilung wurden die Daten als Mittelwert \pm Standardabweichung oder Median (25-/75-Perzentile) dargestellt. Ein Niveau von $p<0.05$ wurde als statistisch signifikant gewertet. 


\section{Ergebnisse}

\subsection{Präoperative Kenngrößen}

\subsubsection{Beschreibung des Patientenkollektivs}

In die Studie wurden die Daten von 26 Patienten eingeschlossen. Alle Patienten wurden postoperativ auf der Intensivstation versorgt.

Jeder der an der Studie teilnehmenden Patienten wurde in der Klinik und Poliklinik für Neurochirurgie des Universitätsklinikums Frankfurts zu einer elektiv geplanten Tumorresektion aufgenommen. Es wurden sowohl maligne Tumore (Astrozytom, Glioblastom) als auch benigne Tumore (Meningeom, Ependymom, Cavernom) reseziert.

\subsubsection{Beschreibung soziodemographischer Merkmale}

Von den 26 in die Studie eingeschlossenen Patienten waren 65\% männlich. Das Durchschnittsalter betrug $58 \pm 13$ Jahre. Das Gewicht betrug $83 \pm 16 \mathrm{~kg}$.

\begin{tabular}{|c|c|}
\hline & Patientenkollektiv \\
\hline $\begin{array}{c}\text { Geschlecht } \\
\text { [männlich] [\%] }\end{array}$ & 65 \\
\hline Alter [Jahre] & $58 \pm 13$ \\
\hline Größe [cm] & $176 \pm 10$ \\
\hline Gewicht [kg] & $83 \pm 16$ \\
\hline BMI [ $\mathbf{k g} / \mathbf{m}^{2}$ ] & $26.8 \pm 4.3$ \\
\hline
\end{tabular}

Tab. 3: Soziodemographische Merkmale.

Die Werte sind als Mittelwert \pm Standartabweichung oder Häufigkeit [\%] angegeben. 


\subsection{Ergebnisse der perioperativen Parameter}

Die präoperativ bestimmten Laborwerte der Routine-Gerinnungsdiagnostik, des Blutbildes und der Retentionsparameter befanden sich bei allen Patienten innerhalb der institutionellen Referenzbereiche für Normwerte.

$N=13$ Patienten unterzogen sich einem Eingriff im Bereich der hinteren Schädelgrube. Mittels transösophagealer Echokardiographie konnte bei jedem dieser Patienten ein persistierendes Foramen Ovale ausgeschlossen werden und die Patienten konnten in halbsitzender Position operiert werden.

Die Zeitspanne zwischen Narkoseeinleitung und Verlegung auf die Intensivstation betrug $326 \pm 118 \mathrm{~min}$. Während dieser Zeit erhielten die Patienten insgesamt $2711 \pm 789 \mathrm{ml}$ kristalloide Infusionslösung. Die in halbsitzender Position operierten Patienten erhielten zusätzlich zwischen den Messzeitpunkten T1 und T2 $1115 \pm 219 \mathrm{ml}$ kolloidale Infusionslösung. Der perioperative Blutverlust betrug $680 \pm 350 \mathrm{ml}$. Die perioperative Urinmenge betrug $1260 \pm 810 \mathrm{ml}$. Keiner der Patienten erhielt zwischen den Messzeitpunkten T1 und T4 allogene Blutprodukte (Erythrozytenkonzentrate, Fresh-Frozen-Plasma, Thrombozytenkonzentrate) oder Gerinnungsfaktorenkonzentrate.

\subsubsection{Thrombelastometrische Auswertung}

In Tabelle 4 und den Abbildungen 5 und 6 sind die Ergebnisse der thrombelastometrischen Untersuchungen dargestellt. Mit Ausnahme der $\mathrm{ML}_{60}$ im EXTEM, waren alle $\mathrm{ML}_{60}$-Werte sowohl im EXTEM- als auch im APTEM-Test gegenüber der Ausgangslage zu T1 signifikant erhöht. 


\begin{tabular}{|c|c|c|c|c|c|c|c|c|}
\hline \multirow{2}{*}{\multicolumn{2}{|c|}{ Parameter }} & T1 & \multicolumn{2}{|c|}{ T2 } & \multicolumn{2}{|c|}{ T3 } & \multicolumn{2}{|l|}{ T4 } \\
\hline & & $\mathrm{n}=\mathbf{2 6}$ & $n=26$ & p & $n=26$ & $p$ & $n=26$ & p \\
\hline \multirow{3}{*}{ EXTEM } & $\mathrm{CT}[\mathrm{s}]$ & $61(49 / 89)$ & $62(47 / 100)$ & 0.230 & $68(51 / 113)$ & 0.039 & $76(60 / 107)$ & 0.199 \\
\hline & $\mathrm{MCF}[\mathrm{mm}]$ & $58(54 / 60)$ & $56(52 / 59)$ & 0.164 & $57(54 / 62)$ & 0.948 & $58(54 / 63)$ & 0.528 \\
\hline & $\mathrm{ML}_{60}[\%]$ & $9(7 / 13)$ & $15(10 / 19)$ & 0.017 & $14(8 / 19)$ & 0.1 & $14(10 / 17)$ & 0.025 \\
\hline FIBTEM & $\mathrm{MCF}[\mathrm{mm}]$ & $12(12 / 18)$ & $9(9 / 13)$ & $<0.001$ & $11(9 / 13)$ & $<0.001$ & $13(10 / 17)$ & 0.441 \\
\hline \multirow{3}{*}{ APTEM } & $\mathrm{CT}[\mathrm{s}]$ & $73(61 / 81)$ & $85(63 / 96)$ & 0.074 & $93(69 / 125)$ & 0.002 & $92(83 / 110)$ & 0.004 \\
\hline & $\mathrm{MCF}[\mathrm{mm}]$ & $58(57 / 63)$ & $57(54 / 58)$ & 0.002 & $57(53 / 62)$ & 0.102 & $56(51 / 61)$ & 0.048 \\
\hline & $\mathrm{ML}_{60}[\%]$ & $10(9 / 12)$ & $14(11 / 19)$ & $<0.001$ & $14(8 / 16)$ & 0.005 & $14(8 / 17)$ & 0.006 \\
\hline
\end{tabular}

Tab. 4: Ergebnisse der thrombelastometrischen Untersuchungen pro Messzeitpunkt (Median, 25/75te Perzentile); p-Werte beschreiben den Unterschied gegenüber der Ausgangslage zu T1. 


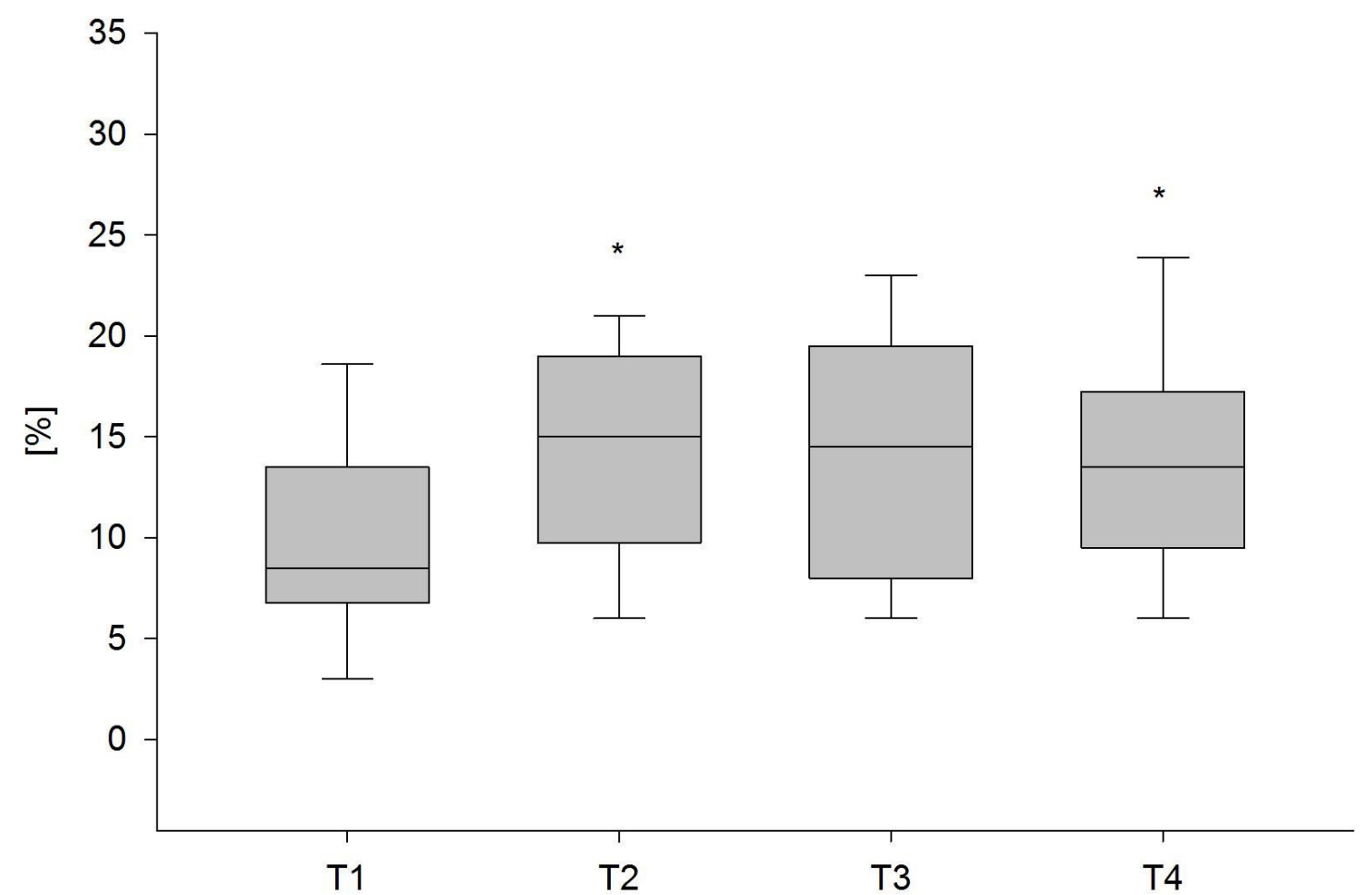

Abb. 5: Veränderung der $\mathrm{ML}_{60}-$ Werte [\%] im EXTEM zwischen den Messzeitpunkten T1-T4. Die Werte sind als Median (25te/75te Perzentile) angegeben; $n=26$; * kennzeichnet $p<0.05$ gegenüber der Ausgangslage zu T1. 


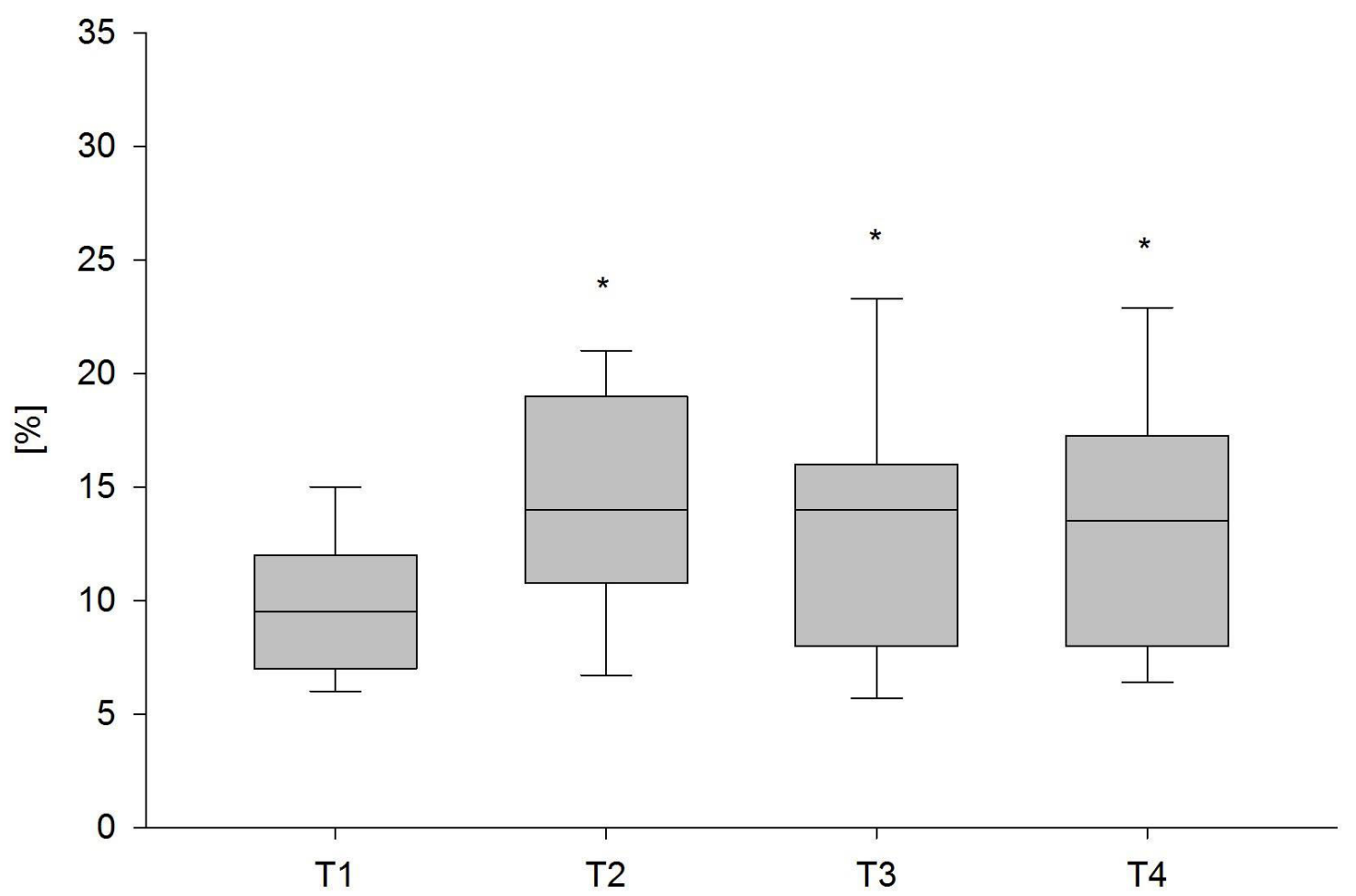

Abb. 6: Veränderung der $\mathrm{ML}_{60}-$ Werte [\%] im APTEM zwischen den Messzeitpunkten T1-T4. Die Werte sind als Median (25te/75te Perzentile) angegeben; $n=26$; * kennzeichnet $p<0.05$ gegenüber der Ausgangslage zu T1.

In Abbildung 7 sind die Faktor XIII-Konzentrationen pro Messzeitpunkt in Relation zu $\mathrm{ML}_{60}$ des APTEM-Tests dargestellt. Außerdem sind die Ergebnisse der Rangsummenkorrelationen pro Messzeitpunkt angegeben. Es konnten zu keinem der vier Messzeitpunkte signifikante Korrelationen zwischen Faktor XIII-Konzentration und der $\mathrm{ML}_{60}$ des APTEM-Tests festgestellt werden. 


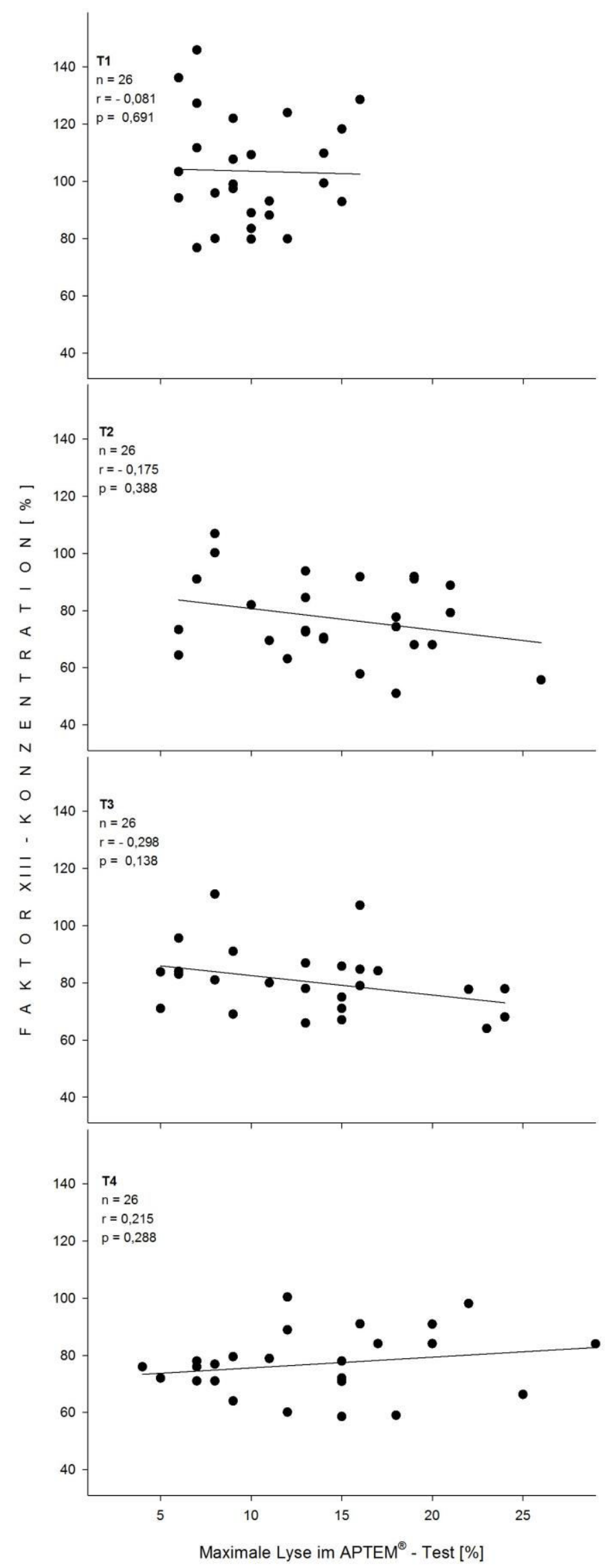

Abb. 7: Streudiagramme mit Regressionskurven zur Darstellung der Korrelationen zwischen der FXIIIKonzentration im Plasma und der maximalen Lyse im APTEM-Test pro Messzeitpunkt $(r=$ SpearmanKorrelationskoeffizient) 


\subsubsection{Konventionelle Gerinnungsdiagnostik und Faktor XIII-Einzelfaktoranalyse}

Die Auswertung der Parameter der konventionellen Gerinnungsdiagnostik und Faktor XIII-Einzelfaktoranalyse zu den vier Messzeitpunkten ist in Tabelle 5 und den Abbildungen 8-12 dargestellt.

Die Fibrinogen-Konzentration sowie die aPTT blieben über die Messungen unverändert gegenüber der Ausgangslage zu T1.

Dagegen waren sowohl Thrombozytenzahl und Hämatokrit als auch die Faktor XIIIKonzentration ab dem Messzeitpunkt T2 signifikant gegenüber der Ausgangslage zu T1 erniedrigt. Der INR-Wert zeigte sich ab T2 gegenüber der Ausgangslage signifikant erhöht.

\begin{tabular}{|c|c|c|c|c|c|c|c|}
\hline \multirow{2}{*}{ Parameter } & T1 & \multicolumn{2}{|c|}{ T2 } & \multicolumn{2}{c|}{ T3 } & \multicolumn{2}{c|}{ T4 } \\
\cline { 2 - 7 } & $\mathbf{n = 2 6}$ & $\mathbf{n = 2 6}$ & $\mathbf{p}$ & $\mathbf{n = 2 6}$ & $\mathbf{p}$ & $\mathbf{n}=26$ & $\mathbf{p}$ \\
\hline $\begin{array}{c}\text { Thrombozyten } \\
{[/ \mathrm{nl}]}\end{array}$ & $256 \pm 73$ & $193 \pm 63$ & $<0.001$ & $201 \pm 62$ & $<0.001$ & $143 \pm 32$ & $<0.001$ \\
\hline $\begin{array}{c}\text { Fibrinogen } \\
{[\mathrm{mg} / \mathrm{dl}]}\end{array}$ & $339 \pm 65$ & $283 \pm 135$ & 0.055 & $285 \pm 147$ & 0.097 & $322 \pm 73$ & 0.239 \\
\hline $\begin{array}{c}\text { Thromboplastinzeit } \\
{[\mathrm{s}]}\end{array}$ & $13.5 \pm 0.5$ & $14.6 \pm 0.8$ & $<0.001$ & $14.6 \pm 0.6$ & $<0.001$ & $14.3 \pm 0.7$ & $<0.001$ \\
\hline $\begin{array}{c}\text { Quick } \\
{[\%]}\end{array}$ & $99 \pm 11$ & $84 \pm 11$ & $<0.001$ & $85 \pm 10$ & $<0.001$ & $88 \pm 10$ & $<0.001$ \\
\hline \begin{tabular}{c} 
INR \\
\hline $\begin{array}{c}\text { aPTT } \\
{[\mathrm{s}]}\end{array}$
\end{tabular} & $1 \pm 0.1$ & $1.1 \pm 0.1$ & $<0.001$ & $1.1 \pm 0.1$ & $<0.001$ & $1.1 \pm 0.1$ & $<0.001$ \\
\hline $\begin{array}{c}\text { Hämatokrit } \\
{[\%]}\end{array}$ & $40 \pm 4$ & $32 \pm 5$ & $<0.001$ & $34 \pm 4$ & $<0.001$ & $35 \pm 3$ & $<0.001$ \\
\hline $\begin{array}{c}\text { FXIII } \\
{[\%]}\end{array}$ & $104 \pm 18$ & $77 \pm 14$ & $<0.001$ & $80 \pm 12$ & $<0.001$ & $77 \pm 11$ & $<0.001$ \\
\hline
\end{tabular}

Tab. 5: Konventionelle Gerinnungslabordiagnostik und FXIII-Einzelfaktorenanalyse pro Messzeitpunkt (Mittelwert \pm Standardabweichung); $p$-Werte beschreiben den Unterschied gegenüber der Ausgangslage zu T1. 


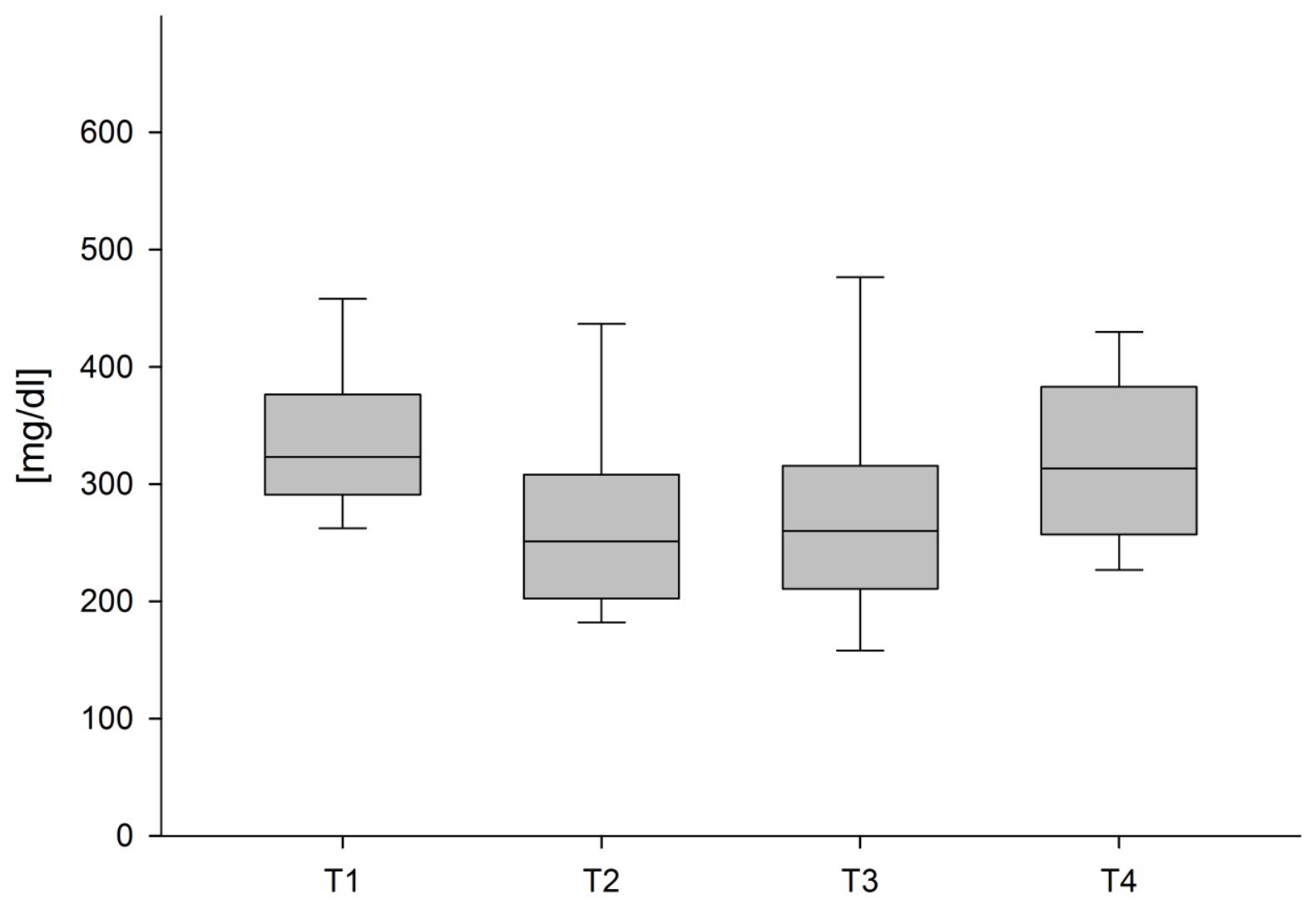

Abb. 8: Veränderung der Fibrinogen-Konzentration [mg/dl] zwischen den Messzeitpunkten T1-T4. Die Werte sind als Median (25te/75te Perzentile) angegeben; $n=26$ 


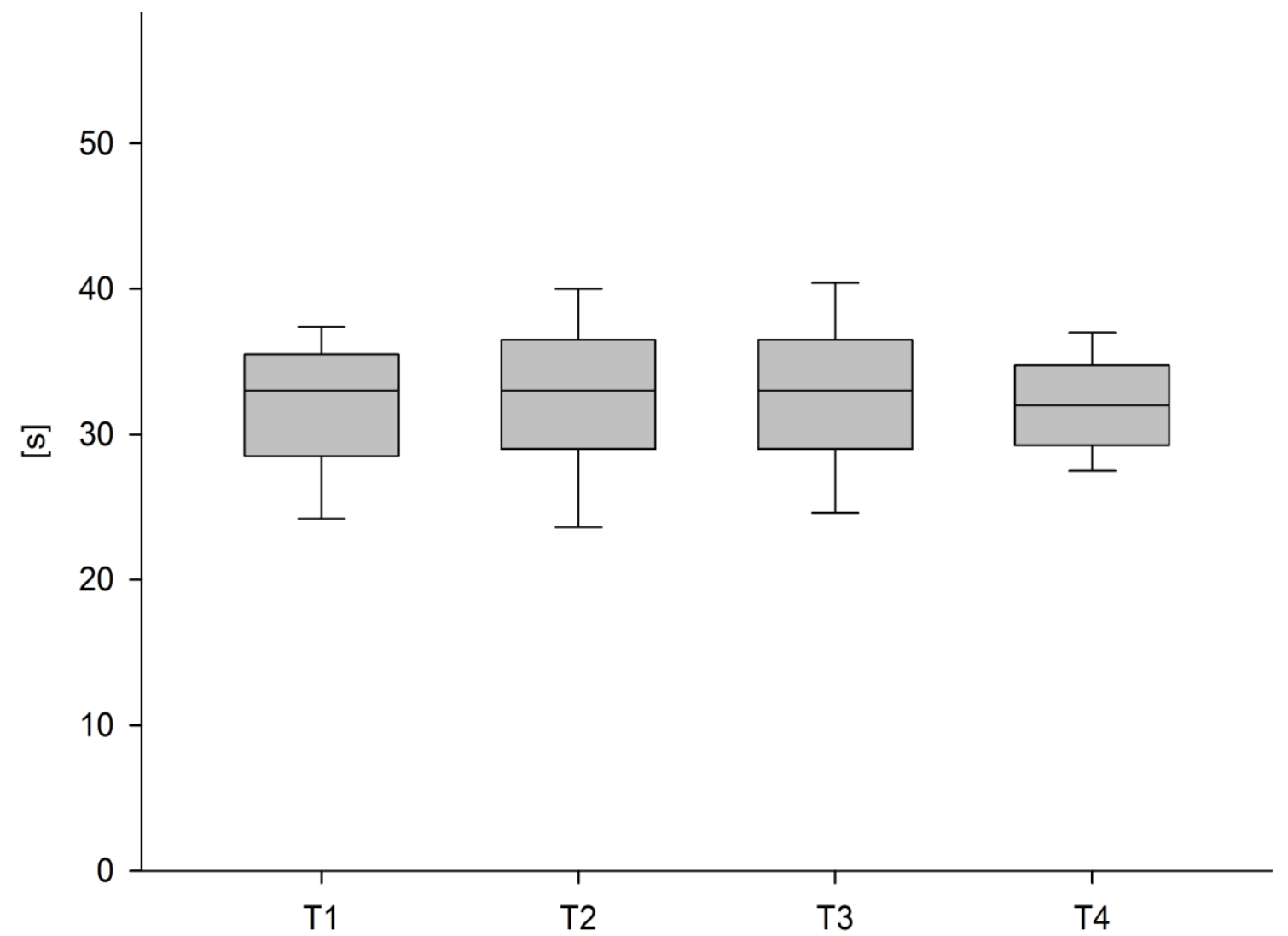

Abb. 9: Veränderung der aPTT [s] zwischen den Messzeitpunkten T1-T4. Die Werte sind als Median (25te/75te Perzentile) angegeben; $n=26$ 


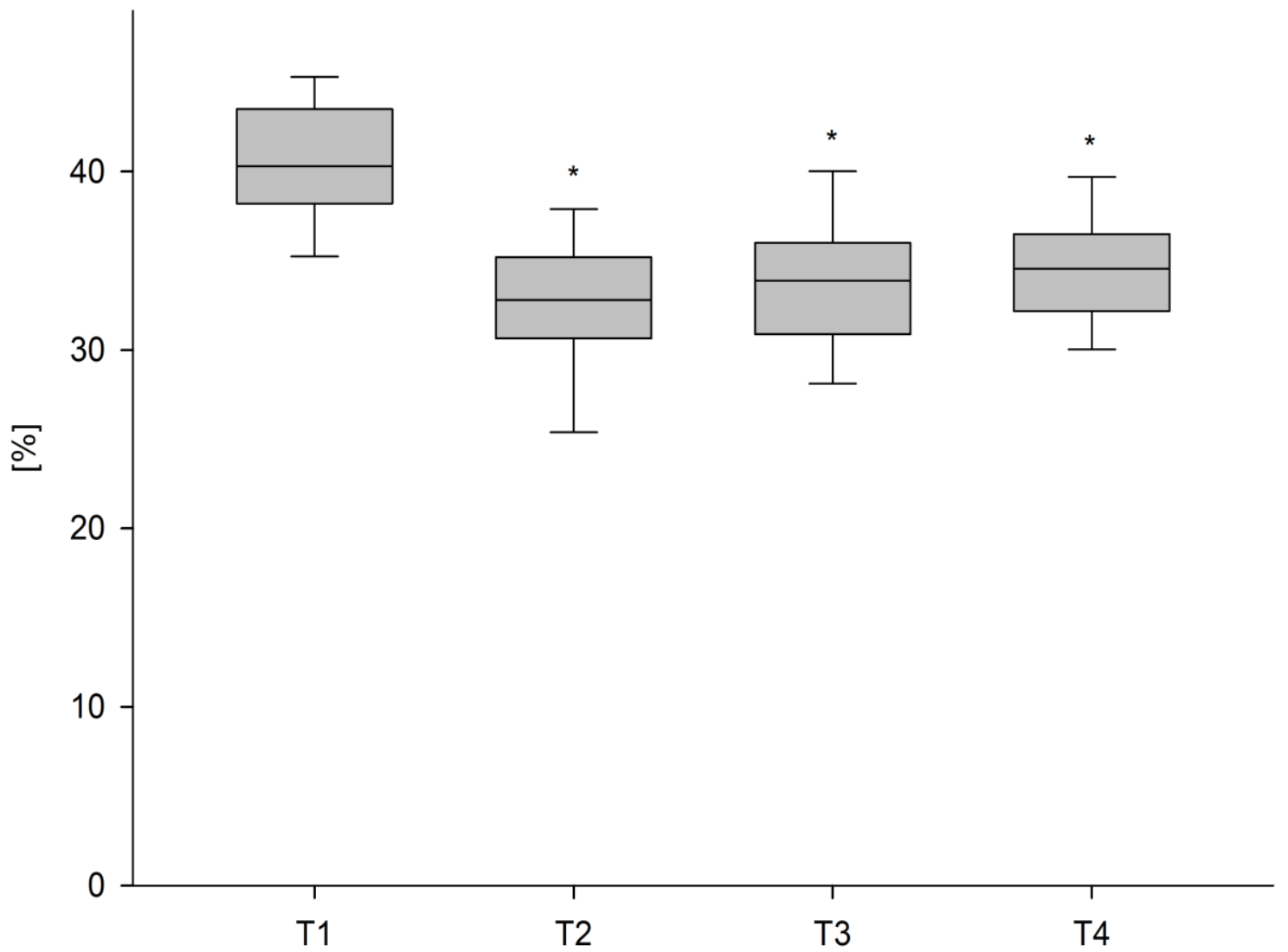

Abb. 10: Veränderung des Hämatokrits [\%] zwischen den Messzeitpunkten T1-T4. Die Werte sind als Median (25te/75te Perzentile) angegeben; $n=26$; * kennzeichnet $p<0.05$ gegenüber der Ausgangslage zu T1. 


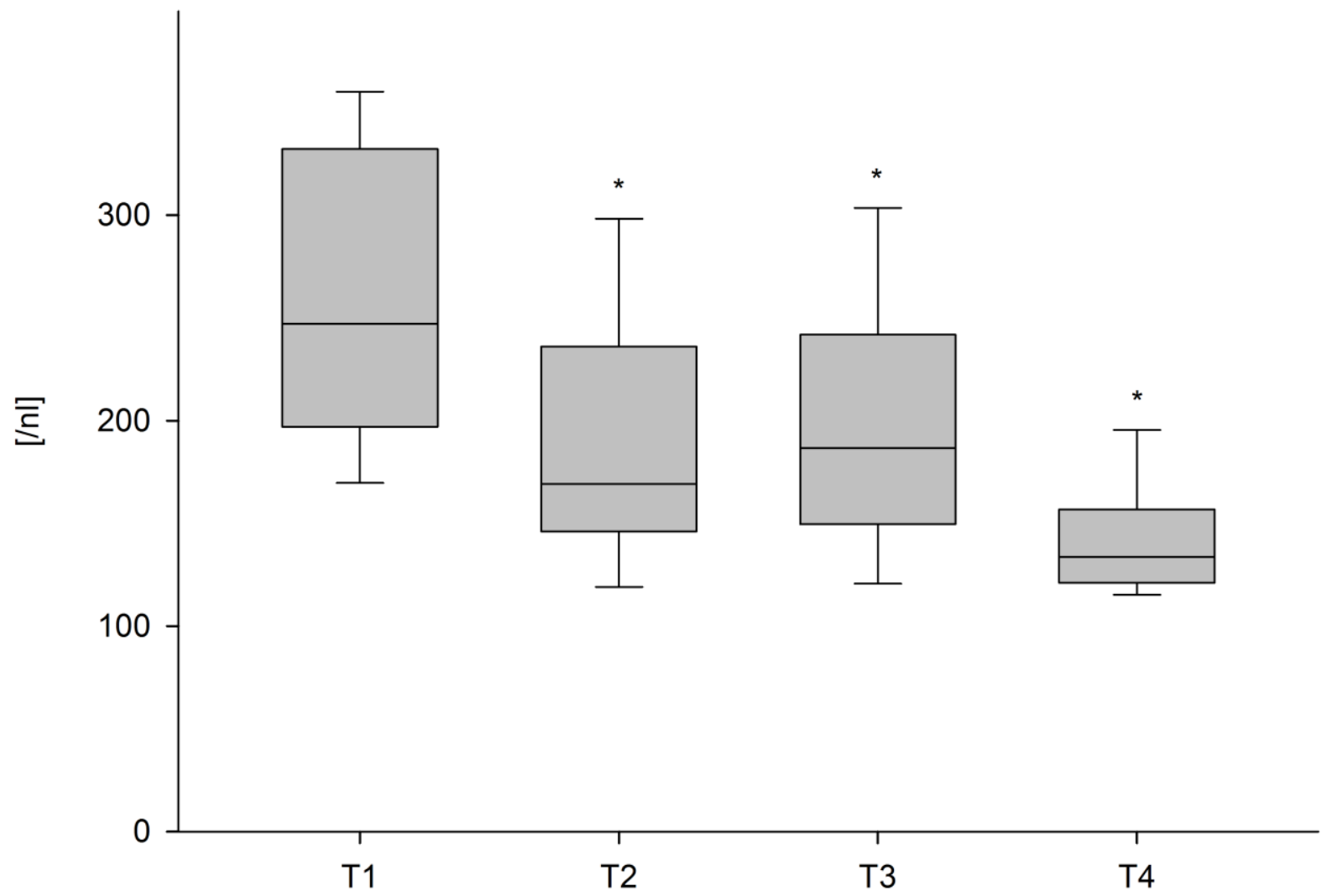

Abb. 11: Veränderung der Thrombozyten-Konzentration [/nl] zwischen den Messzeitpunkten T1-T4. Die Werte sind als Median (25te/75te Perzentile) angegeben; $n=26$; ${ }^{*}$ kennzeichnet $p<0.05$ gegenüber der Ausgangslage zu T1. 


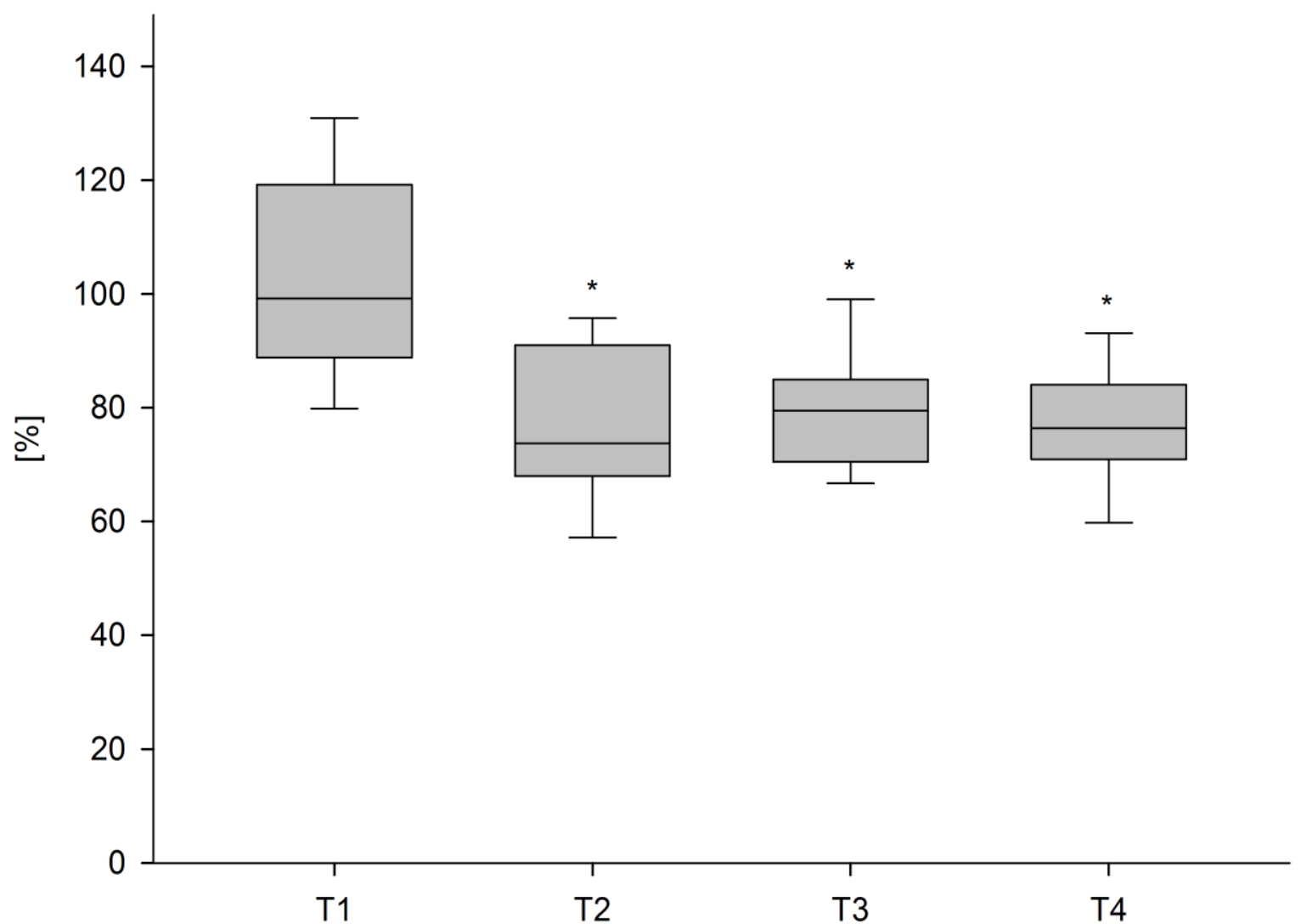

Abb. 12: Veränderung der Faktor XIII-Konzentration [\%] zwischen den Messzeitpunkten T1-T4. Die Werte sind als Median (25te/75te Perzentile) angegeben; $n=26$; * kennzeichnet $p<0.05$ gegenüber der Ausgangslage zu T1. 


\subsection{Multiple lineare Regressionsanalyse}

Die Ergebnisse der multiplen linearen Regressionsanalyse sind in Tabelle 6 dargestellt. Es zeigte sich, dass weder die Thrombozytenzahl noch die Fibrinogen-oder FXIII-Konzentration signifikante Prädiktoren für die $\mathrm{ML}_{60}$ des APTEM-Tests waren.

\begin{tabular}{|c|c|c|c|c|}
\hline \multicolumn{2}{|c|}{} & Koeffizient & Standardfehler & $\mathbf{p}$ \\
\hline \multirow{4}{*}{ T1 } & Thrombozyten & 0.0134 & 0.0086 & 0.136 \\
\cline { 2 - 5 } & Fibrinogen & -0.0053 & 0.0099 & 0.594 \\
& Faktor XIII & 0.0075 & 0.03391 & 0.827 \\
\hline \multirow{4}{*}{ T2 } & Thrombozyten & 0.0033 & 0.0176 & 0.853 \\
\cline { 2 - 5 } & Fibrinogen & 0.0022 & 0.0082 & 0.791 \\
& Faktor XIII & -0.1021 & 0.0757 & 0.193 \\
\hline \multirow{3}{*}{ T3 } & Thrombozyten & 0.0099 & 0.0199 & 0.621 \\
\cline { 2 - 5 } & Fibrinogen & 0.0073 & 0.0084 & 0.397 \\
& Faktor XIII & -0.1771 & 0.0961 & 0.080 \\
\hline \multirow{3}{*}{ T4 } & Thrombozyten & -0.0159 & 0.0417 & 0.707 \\
\cline { 2 - 5 } & Fibrinogen & 0.0316 & 0.0168 & 0.073 \\
& Faktor XIII & 0.1281 & 0.1161 & 0.282 \\
\hline
\end{tabular}

Tab. 6: Multiple lineare Regressionsanalyse zur Analyse potentieller Prädiktoren für die $\mathrm{ML}_{60}$ des APTEM-Tests zu jedem Messzeitpunkt. Als unabhängige Variablen wurden die Thrombozytenzahl sowie die Fibrinogen- und FXIII-Konzentration definiert. 


\section{Diskussion}

Das Hauptergebnis dieser Arbeit zeigte, dass die Faktor XIII-Konzentration zu keinem der vier Messzeitpunkte mit dem Ausmaß der maximalen Lyse nach 60 Minuten $\left(\mathrm{ML}_{60}\right)$ im APTEM-Test des ROTEM ${ }^{\circledR}$ korrelierte [39]. Anders als Fallberichte, unter anderem auch aus unserer Arbeitsgruppe [33], vermuten ließen, zeigte sich kein Zusammenhang zwischen der $\mathrm{ML}_{60}$ und der Faktor XIIIKonzentration.

Von Ergebnissen der Routine-Gerinnungsdiagnostik wie z.B. aPTT oder INR kann nicht auf die Plasmakonzentration von Faktor XIII geschlossen werden. Besonders bei Patienten, die sich einem neurochirurgischen Eingriff unterziehen, können intrasowie postoperative Blutungen schwerwiegende Komplikationen darstellen. Die Inzidenz von postoperativen Nachblutungen bei neurochirurgischen Patienten wird in der Literatur sehr unterschiedlich beschrieben [1]. Sie reicht von $0,8 \%$ bis $50 \%$ [1113]. In einer Studie der Universität Oslo mit einem Patientenkollektiv von $\mathrm{n}=2630$ Patienten wurden Mortalität und Komplikationen nach Kraniotomien untersucht. Mit $35 \%$ stellten postoperativ aufgetretene Hämatome die Haupttodesursache nach Kraniotomie dar. Insgesamt mussten sich $2.1 \%$ des Patientenkollektivs einer Rekraniotomie auf Grund einer postoperativen Blutungskomplikation unterziehen [40]. Als Risikofaktoren für eine erhöhte postoperative Mortalität bzw. Komplikationsrate werden neben hohem Alter, erniedrigtem perioperativen Karnofsky Score und Komorbidität insbesondere das Auftreten einer disseminierten intravasalen Koagulopathie sowie eines Faktor XIII-Mangels beschrieben [1, 41, 42].

Gerlach et al. zeigten in ihrer Studie an einem großen Patientenkollektiv $(n=1264)$, dass es bei $1.6 \%(n=20)$ der Patienten nach neurochirurgischer Operation zu schwerwiegenden Blutungen kam. Die Faktor XIII-Konzentration wurde hier bei 34 Patienten untersucht, bei denen eine Gerinnungsstörung vermutet wurde (thromboembolische Ereignisse, schwere Blutungen, Wundhämatom). Man fokussierte auf eine Gruppe von Patienten mit erniedrigter FXIII-Konzentration $(<60 \%)$ und konnte zeigen, dass jeder dieser Patienten eine schwerwiegende postoperative Blutung entwickelte, die bei $75 \%(n=6)$ eine Rekraniotomie notwendig machte [20]. Die Autoren folgerten, dass eine verminderte Faktor XIII-Aktivität mit 
einem erhöhten Risiko postoperativer Blutungen nach neurochirurgischen Eingriffen korrelieren könnte [20].

Faktor XIII ist ein Gerinnungsfaktor, der am Ende der Gerinnungskaskade die aus Fibrin-Monomeren bestehenden, löslichen Fibrinstränge quervernetzt, um so ein stabiles, unlösliches Fibringerinnsel zu bilden [33, 43]. In seiner aktivierten Form (FXIIla) katalysiert Faktor XIII die Bildung kovalenter Bindungen zwischen der $\gamma$-Carbamid-Gruppe des Glutamins und der $\varepsilon$-Amin-Gruppe des Lysins unter Abspaltung von Ammoniak. Diese Quervernetzung sorgt für eine mechanische Stabilisierung des vorgeformten Fibringerinnsels [43]. Neben dieser gerinnselstabilisierenden Eigenschaft verankert FXIII sowohl Fibrinogen als auch alpha-2-Antiplasmin innerhalb des Fibringerinnsels. Damit stellt FXIII auf der einen Seite das Hauptsubstrat der Gerinnselformation zur Verfügung und sorgt auf der anderen Seite für eine gewisse antifibrinolytische Widerstandskraft [33].

Ein Faktor XIII-Defizit kann zu schweren Blutungskomplikationen führen. Man unterscheidet grundsätzlich das hereditäre von dem erworbenen Faktor XIII-Defizit. Das hereditäre Faktor XIII-Defizit zählt mit einer Prävalenz von circa $1: 4.000 .000 \mathrm{zu}$ den seltensten Gerinnungsstörungen, ist aber auf Grund seines Schwergrades an Blutungserscheinungen Gegenstand intensiver klinischer und genetischer Studien [43]. Als Symptome sind intrazerebrale Blutungen im Kindesalter, erhöhte Blutungsneigung bei Bagatelltraumata, Wundheilungsstörungen und die Neigung zu Spontanabort beschrieben [44-47].

Deutlich häufiger zeigt sich bei perioperativen Blutungskomplikationen ein vorwiegend durch Verbrauchskoagulation (z.B. disseminierte intravasale Koagulopathie) oder Dilution bedingtes erworbenes Faktor XIII-Defizit [33, 43, 48].

In einer Studie an einem kardiochirurgischen Kollektiv stellte sich die FXIIIKonzentration als unabhängiger Prädiktor für den postoperativen Blutverlust dar [49]. Fallberichte und in-vitro-Studien legen die Vermutung nahe, dass sich die Thrombelastometrie als Verfahren zum Monitoring der Faktor XIII-Konzentration eignen könnte. Eine in-vitro diagnostizierte Aprotinin-resistente Lyse könnte demnach als Ausdruck nicht fibrinolytisch bedingter verminderter Gerinnselstabilität mit der FXIII-Konzentration korrelieren [33-35, 39].

Jámbor et al. konnten ex-vivo eine Konzentrations- und Wirkbeziehung zwischen FXIII-Konzentration und MCF-, CFT-, und ML-Wert im ROTEM ${ }^{\circledR}$ nachweisen. Sie hemmten in ihrer Studie Faktor XIII durch einen polyklonalen, neutralisierenden 
Antikörper (NAB) und führten eine thromboelastometrische Untersuchung von aktiviertem Vollblut (mit gehemmten Blutplättchen) durch. Im EXTEM-Test zeigte sich die CFT verlängert, die MCF herabgesetzt und die fibrinolytischen Effekte ausgeprägter als in der Vergleichsgruppe ohne spezifische Faktor XIII-Hemmung [39, 50]. Der Einfluss von Faktor XIII auf die Clotstabilität konnte außerdem von Theusinger et al. beschrieben werden, die nach in-vitro-Substitution von Faktor XIII eine signifikante Zunahme der Clotfestigkeit in EXTEM- und APTEM-Test sowie eine Reduktion der ML im EXTEM-Test beobachten konnten [35]. Einschränkend muss allerdings bei der Interpretation der Ergebnisse dieser Studie berücksichtigt werden, dass die in-vitro-Substitution von 0,32 IU FXIII, 0,63 IU FXIII und 1,25 IU FXIII, zu unphysiologisch hohen FXIII-Konzentration von 150, 300 und $600 \%$ führte und der beschriebene Effekt damit klinisch nur von untergeordneter Relevanz ist.

Auch in weiteren Studien wurde die Hypothese aufgestellt, dass sich die Thrombelastometrie, in der die Gerinnselstabilität sowie dessen Lyse über die Zeit graphisch aufgetragen werden, als Verfahren zum Nachweis eines Faktor XIIIMangels eignen könnte [33, 34, 37, 39, 51-53].

In thrombelastometrischen Ringversuchen konnten Dick et al. eine gute Reproduzierbarkeit der mittels ROTEM ${ }^{\circledR}$ erzielten Messergebnisse nachweisen [54]. Besonders bei perioperativer Koagulopathie wäre ein bettseitiges Verfahren zur Diagnostik eines Faktor XIII-Mangels vorteilhaft, denn es könnte dabei behilflich sein die Indikation zur Transfusion von FFP bzw. zur Applikation von Faktorenkonzentraten zu untermauern. Verglichen mit den Ergebnissen der konventionellen Gerinnungsdiagnostik, liegen die für effiziente Hämotherapie notwendigen thrombelastometrischen Messergebnisse nach deutlich kürzerer Zeit vor [32, 55].

Für den Einsatz als perioperativ einsetzbares Point-of-Care-Verfahren ist die Benutzerfreundlichkeit des jeweiligen Systems von großer Bedeutung. Auch Mitarbeiter ohne besondere laboratoriumsmedizinische Vorkenntnisse müssen in der Lage sein, schnelle und valide Ergebnisse zu erzielen. Haas und Kollegen verglichen in diesem Zusammenhang die ROTEM ${ }^{\circledR}$-Analysen von 90 Blutproben pädiatrischer Patienten, die einerseits durch einen einzelnen geschulten Mitarbeiter innerhalb des Operationssaals durchgeführt wurde, und andererseits durch ausgebildetes Laborpersonal im klinikeigenen Labor ausgewertet wurden. Im Ergebnis zeigten sich lediglich geringe Unterschiede in den ROTEM ${ }^{\circledR}$-Untersuchungen beider Gruppen 
(unabhängig davon ob diese durch OP-Mitarbeiter oder Laborpersonal durchgeführt wurden). Gleichzeitig kam es zu einer deutlichen Zeitersparnis (11 Minuten) zugunsten der bettseitigen Auswertung [56].

In zwei prospektiven Observationsstudien benötigte das Zentrallabor für die Auswertung der $\mathrm{zu}$ untersuchenden Blutproben im Mittel $88 \mathrm{~min}$ (Streuung: 29-235 $\mathrm{min}$ ) beziehungsweise $53 \mathrm{~min}$ (Streuung: 45-63 $\mathrm{min}$ ) [32, 57, 58]. Die Ergebnisse aus dem A-10 Test des ROTEM ${ }^{\circledR}$ standen bereits im Mittel nach 23 min (Streuung: 21-24 min) zur Verfügung [32]. Wird das ROTEM ${ }^{\circledR}$ als bettseitiges Verfahren genutzt, ergibt sich auf Grund der entfallenen Transportwege der Blutproben eine weitere Zeitersparnis von etwa 5 Minuten gegenüber der konventionellen Gerinnungsdiagnostik [57].

Neben dem ROTEM ${ }^{\circledast}$ findet mit dem TEG ${ }^{\circledR}$ (Haemonetics Corp., Niles, IL), das auf einer vergleichbaren Methodik basiert, ein weiteres Gerät breite klinische Anwendung als POC-Verfahren. Im direkten Vergleich beider Systeme liegen bei dem mittels "tissue factor" aktivierten ROTEM ${ }^{\circledast}$ die ersten verwertbaren Ergebnisse zur Clotbildung (CT und CFT vs. R-time und K-time) bereits 7-9 min früher vor als bei dem TEG ${ }^{\circledR}$, welches durch Kaolin aktiviert wird $[57,59]$.

Mehrere Arbeitsgruppen konnten zeigen, dass durch den Einsatz des ROTEM $^{\circledR}$ als POC-Verfahren Transfusionsraten von EK, FFP und TK deutlich geringer, die postoperative Beatmungszeit sowie der Aufenthalt auf der Intensivstation kürzer, unerwünschte Ereignisse seltener, die Kosten der hämostatischen Therapie weniger und die 6-Monatsmortalität niedriger waren als in einem vergleichbaren Kollektiv ohne POC Messungen [57, 60].

Auf der Grundlage klinikinterner "Standard Operating Procedures" (SOP) wurde die periprozedurale Infusionstherapie auch unter Verwendung kolloidaler Infusionslösung (HES) durchgeführt. Volumensubstitution im Allgemeinen führt dilutionsbedingt zu einer relativen Verminderung der Konzentration von im Blut vorhandenen Gerinnungsfaktoren und Thrombozyten. Aktuelle Studien lassen darauf schließen, dass kolloidale Infusionslösungen die Fibrinpolymerisation signifikant beeinflussen, die Thrombozytenfunktion behindern und die Konzentration von Faktor VIII und Von-Willebrand-Faktor herabsetzen [61-66].

Schramko und Kollegen untersuchten in diesem Zusammenhang den Einfluss kolloidaler Infusionslösungen (Hydroxyethylstärke und Gelatine) auf die Blutgerinnung nach Herzoperationen. 45 Patienten erhielten drei Boli (je $7 \mathrm{ml} / \mathrm{kg}$ ) von 
entweder 6\% HES 130/0,4, 4\% Gelatine oder Ringer-Acetat Lösung nach elektiver Herzoperation. Die Infusion der jeweiligen Studienlösung in der Dosierung $7 \mathrm{ml} / \mathrm{kg}$ wurde über die folgenden 12 Stunden fortgesetzt. Die Gesamtdosis der jeweiligen Studienlösung betrug $28 \mathrm{ml} / \mathrm{kg}$. Hypovolämien wurden mittels Ringer-Acetat Lösung ausgeglichen. Anschließend wurde eine modifizierte thrombelastographische Untersuchung mittels ROTEM ${ }^{\circledR}$ durchgeführt.

Nach der Substitution von 7, 14 und $21 \mathrm{ml} / \mathrm{kg}$ kolloidaler Infusionslösungen kam es zu einer Verlängerung der CFT und einer Verminderung der MCF gegenüber der Vergleichsgruppe, die Ringer-Acetat Lösung erhielt. Am ersten postoperativen Morgen zeigte sich die MCF in der Gelatinegruppe immer noch herabgesetzt im Vergleich zu der Patientengruppe die Ringer-Acetat Lösung erhielt. Die HES-Gruppe hingegen wies Normalwerte bezüglich der MCF auf. Letztlich zeigt die Studie, dass selbst kleine Mengen HES 130/0,4 oder Gelatine die MCF dosisabhängig vermindern [67].

Nielsen und Mitarbeiter konnten außerdem nachweisen, dass eine HES induzierte Dilution die Wahrscheinlichkeit einer Fibrinolyse erhöht, da es zu einer Verminderung der alpha-2-Antiplasmin-Plasmin Interaktion kommt [68].

Um etwaige Kolloid-assoziierte-Koagulopathien zu detektieren, wurde in der vorliegenden Studie der Messzeitpunkt T4 $24 \mathrm{~h}$ nach postoperativer Verlegung auf die Intensivstation definiert. Wie bereits erläutert, ist anzunehmen, dass eine transfusionsassoziierte Dilution zu einer Verringerung der FXIII-Konzentration führt. Ein MZP T4 zu einem späteren Zeitpunkt wäre daher aussagekräftig gewesen. Da dies aber nicht Ziel unserer Studie war, wurde darauf verzichtet.

Als primäre Zielvariable wurde die maximale Lyse nach 60 Minuten im APTEM-Test des ROTEM ${ }^{\circledR}$ gewählt $\left(\mathrm{ML}_{60}\right)$.

Ebenso wie das Hämostasesystem unterliegt auch das Fibrinolysesystem einer sensiblen Kontrolle. Unter physiologischen Bedingungen wird die Thrombolyse hauptsächlich durch "tissue plasminogen factor" (t-Pa) gesteuert, welcher Plasminogen in Plasmin umwandelt und so zu einem Zerfall des Fibrinnetzwerkes, der Fibrinolyse, führt. Im Falle von großen Operationen, Trauma oder Schock kann es durch Schädigung der inhibitorischen Mechanismen oder überschießender Plasminbildung $\mathrm{zu}$ einem Ungleichgewicht zwischen Gerinnselbildung und Fibrinolyse zu Gunsten der Fibrinolyse kommen [69]. Die systemische Aktivierung der Fibrinolyse wird als Hyperfibrinolyse bezeichnet und kann durch Auflösung von 
Gerinnseln und die Zerstörung des zirkulierenden Fibrinogens zu einer lebensbedrohlichen Blutung führen [70]. In unserer Studie wurde eine Hyperfibrinolyse definiert als die Verringerung der MCF im ROTEM ${ }^{\circledR}$ von mehr als 15\% nach 60 Minuten.

Durch die in-vitro-Zugabe des Antifibrinolytikums Aprotinin (APTEM-Test im ROTEM $^{\circledR}$ ) würde eine Plasmin-assoziierte Hyperfibrinolyse in-vitro gehemmt werden. Aprotinin ist ein unspezifischer Serin-Protease-Hemmer, der aus der Rinderlunge gewonnen wird und Plasmin durch Komplexbildung hemmt. Die Spaltung des Fibrinmoleküls wird so blockiert und die Fibrinolyse verlangsamt [70].

Eine erhöhte Lyse $\left(\mathrm{ML}_{60}>15 \%\right)$ im APTEM-Test kann folglich nicht Plasmin assoziiert sein, sondern ist am ehesten auf eine verminderte Gerinnselstabilität zurückzuführen. Da Faktor XIII als gerinnselstabilisierender Faktor die Gerinnselstärke maßgeblich mitbeeinflusst, lässt dies den Schluss zu, dass sich ein Faktor XIII - Defizit durch die Lyse im APTEM - Test des ROTEM ${ }^{\circledR}$ detektieren lässt. In Abbildung 7 sind zu jedem Messzeitpunkt die Faktor XIII-Konzentrationen und die entsprechende $\mathrm{ML}_{60}$ im APTEM-Test aufgetragen [39].

Die Ergebnisse unserer Studie zeigten, dass bei $\mathrm{ML}_{60}$-Werten $>15 \% \mathrm{zu}$ den Messzeitpunkten T2, T3 und T4 zum Teil Faktor XIII-Konzentrationen > 70-80\% vorlagen.

Das bedeutet, dass die $\mathrm{ML}_{60}$ im APTEM-Test zumindest nicht ausschließlich durch ein Faktor XIII-Defizit zu erklären ist [39]. Die Gerinnselstabilität wird im Wesentlichen durch Thrombozyten, Fibrinogen und Faktor XIII bestimmt. Als potentielle Prädiktoren für die $\mathrm{ML}_{60}$ im APTEM-Test führten wir eine multiple lineare Regressionsanalyse mit den Hauptdeterminanten der Gerinnselstabilität als unabhängige Variablen durch (Tabelle 6). Hier konnte sich keiner der drei Faktoren als signifikanter Prädiktor für die $\mathrm{ML}_{60}$ herausstellen [39].

Die Analyse der soziodemographischen Daten zeigt, dass es weder Unterschiede in der präoperativen antikoagulatorischen Medikation, noch in der Anzahl der perioperativ transfundierten allogenen Blutprodukte bzw. der substituierten Gerinnungsfaktoren-Konzentrate gab. Es konnten keine Unterschiede zwischen der konventionellen Gerinnungsanalyse und den Rahmenbedingungen der Hämostase wie dem pH-Wert, der Calcium-Konzentration, der Körpertemperatur oder des Hämatokrits zu den Messzeitpunkten T1 und T2 festgestellt werden. Außerdem 
zeigte die Auswertung der Ergebnisse keine Differenzen in der Art des operativen Eingriffs oder der bestehenden Vorerkrankungen.

\section{Limitationen}

Die Hauptlimitation der vorliegenden Studie stellt die geringe Größe des Patientenkollektivs dar. Eine im Vorfeld durchgeführte Fallzahlanalyse basierte auf der zu erwartenden Veränderung in der Konzentration von Faktor XIII, nicht aber auf Veränderung der primären Zielvariablen $\mathrm{ML}_{60}$ im APTEM-Test [33].

Zum Zeitpunkt der Studienplanung existierten keine Daten über den perioperativen Verlauf der $\mathrm{ML}_{60}$. Damit gab es keine Alternative zu Faktor XIII als Grundlage zur Fallzahlanalyse. Erst während der Studiendurchführung wurde eine relativ große Standardabweichung der $\mathrm{ML}_{60}$ offensichtlich $(10 \pm 3 \%$ an $\mathrm{T} 1,15 \pm 5 \%$ an $\mathrm{T} 2$, $13 \pm 6 \%$ an T3 und $14 \pm 6 \%$ an T4). Wäre diese erhöhte Standardabweichung berücksichtigt worden, hätte sich in der Fallzahlanalyse ein größeres Patientenkollektiv ergeben. Des Weiteren zeigten sich bei lediglich 24 der insgesamt 104 (23\%) bestimmten Faktor XIII-Konzentrationen Werte < 70\%. Wäre die Anzahl der Patienten mit klinisch relevantem Faktor XIII-Mangel größer gewesen, hätte dies die Aussagekraft der durchgeführten Untersuchung positiv beeinflussen können. Die Ergebnisse könnten daher von statistischen Fehlern 1. und 2. Art betroffen sein.

Eine weitere Limitation findet sich in dem heterogenen Patientenkollektiv. Da ein Teil der Patienten in halbsitzender Position operiert wurde, erhielten diese auf Grund klinikinterner Behandlungsalgorithmen zwischen T1 und T2 zusätzlich $15 \mathrm{ml} / \mathrm{kg}$ Körpergewicht kolloidale Infusionslösung [33]. Ein relevanter Einfluss der kolloidalen Lösung auf die $\mathrm{ML}_{60}$ durch Dilution ist möglich. In perioperativen Blutungssituationen ist die Substitution von kolloidalen Infusionslösungen ein Standardverfahren. In unsere Studie wurden $\mathrm{n}=13$ Patienten eingeschlossen, denen perioperativ kolloidale Infusionslösungen infundiert wurden. Eine Ausweitung der Fragestellung nach dem Stellenwert der Thrombelastometrie für das Monitoring von Faktor XIII auf dieses Patientenkollektiv wäre grundsätzlich sinnvoll. Die Größe des betreffenden Patientenkollektivs ( $n=13$ ) erscheint allerdings zu gering, um statistisch signifikante Ergebnisse erzielen zu können.

In ihrer Zusammenschau können die Ergebnisse der durchgeführten Untersuchung nicht bestätigen, das ein Zusammenhang zwischen der $\mathrm{ML}_{60}$ im APTEM-Test des ROTEM $^{\circledR}$ und der Faktor XIII-Konzentration besteht. Die aufgeführten methodischen 
Limitationen dieser Studie lassen endgültige Aussagen bezüglich der Eignung der Thrombelastometrie für das Monitoring eines potentiellen Faktor XIII-Mangels nicht zu. 


\section{Zusammenfassung}

\section{STUDENZIEL}

Untersuchungen legen nahe, dass eine erhöhte maximale Lyse im APTEM-Test $\left(\mathrm{ML}_{60}>15 \%\right)$ des ROTEM ${ }^{\circledR}$ (Pentapharm GmbH, München) ein FXIII-Defizit (FXIII < $70 \%)$ anzeigen könnte. In dieser Studie sollte untersucht werden, ob sich thrombelastometrische Messungen eignen, um den isolierten Einfluss von FXIII auf die Gerinnselstabilität wiederzugeben und damit ein potenzielles FXIII-Defizit anzuzeigen.

\section{METHODEN}

Nach positivem Ethikvotum nahmen 26 Patienten, die sich einer elektiv geplanten Kraniotomie zur Tumorresektion unterzogen, an der Studie teil. Unmittelbar nach Einleitung einer Allgemeinanästhesie (T1), vor Hautschnitt (T2), bei (T3) und $24 \mathrm{~h}$ nach (T4) postoperativer Aufnahme auf die Intensivstation wurden Blutentnahmen für konventionelle Gerinnungsanalysen, FXIII-Einzelfaktoranalysen und thrombelastometrische Untersuchungen mit dem ROTEM ${ }^{\circledR}$-System (EXTEM-, FIBTEM- und APTEM-Test) entnommen. Zur statistischen Analyse wurden Rangsummenkorrelationen nach Spearman und multiple lineare Regressionsanalysen durchgeführt.

\section{ERGEBNISSE}

Die FXIII-Konzentrationen korrelierten zu keinem Messzeitpunkt mit der $\mathrm{ML}_{60}$ im APTEM-Test. Weder Thrombozytenzahl noch Fibrinogen- oder FXIII-Konzentration besaßen einen prädiktiven Wert für die $\mathrm{ML}_{60}$ des APTEM-Tests.

\section{SCHLUSSFOLGERUNG}

Die Ergebnisse dieser Untersuchung sprechen nicht dafür, dass die Thrombelastometrie im gewählten Kollektiv zum perioperativen Monitoring der FXIIIKonzentration geeignet sein könnte. 


\section{Summary}

\section{OBJEKTIVE}

Studies give evidence that an increased maximum lysis in the APTEM-test $\left(\mathrm{ML}_{60}>15 \%\right)$ of the ROTEM ${ }^{\circledR}$ (Tem International GmbH, Munich, Germany) might indicate a factor XIII deficiency (FXIII $<70 \%$ ). It was the aim of this study to investigate the feasibility of thrombelastometric measurements with the ROTEM ${ }^{\circledR}$ device to reflect the isolated influence of FXIII on clot stability and therefore to indicate potential factor XIII deficiencies.

\section{METHODS}

After approval by the local Scientific and Ethic Review Board, 26 consecutive patients, scheduled for elective craniotomy for tumor resection, were prospectively enrolled into this study. Blood samples were taken for conventional laboratory coagulation analyses, FXIII analyses and thrombelastometric measurements (EXTEM, FIBTEM and APTEM tests) after induction of general anaesthesia (T1), before skin incision (T2) as well as at (T3) and 24 hours after (T4) postoperative admission to ICU, respectively. Statistical analyses included Spearman rank order correlations and multiple linear regressions.

\section{RESULT}

FXIII concentrations did not correlate with the $\mathrm{ML}_{60}$ in the APTEM-test at any measuring point. Neither platelet count nor fibrinogen nor FXIII concentrations were of predictive value for $\mathrm{ML}_{60}$ of the APTEM-test.

\section{CONCLUSION}

The results lead to the assumption that thrombelastometric measurements may not be appropriate for the perioperative monitoring of FXIII concentration. 


\section{Literaturverzeichnis}

1. Seifman, M.A., et al., Postoperative intracranial haemorrhage: a review. Neurosurg Rev, 2011. 34(4): 393-407.

2. Stanworth, S.J., et al., Which groups of patients are transfused? A study of red cell usage in London and southeast England. Vox Sang, 2002. 83(4): 352-7.

3. Weber, C.F., [Modern coagulation management reduces the transfusion rate of allogenic blood products]. Anasthesiol Intensivmed Notfallmed Schmerzther, 2012. 47(6): 418-24.

4. Vivacqua, A., et al., Morbidity of bleeding after cardiac surgery: is it blood transfusion, reoperation for bleeding, or both? Ann Thorac Surg, 2011. 91(6): 1780-90.

5. Spiess, B.D., et al., Platelet transfusions during coronary artery bypass graft surgery are associated with serious adverse outcomes. Transfusion, 2004. 44(8): 1143-8.

6. Marik, P.E. and H.L. Corwin, Efficacy of red blood cell transfusion in the critically ill: a systematic review of the literature. Crit Care Med, 2008. 36(9): 2667-74.

7. Murphy, G.J., et al., Increased mortality, postoperative morbidity, and cost after red blood cell transfusion in patients having cardiac surgery. Circulation, 2007. 116(22): 2544-52.

8. Dara, S.I., et al., Fresh frozen plasma transfusion in critically ill medical patients with coagulopathy. Crit Care Med, 2005. 33(11): 2667-71.

9. Glance, L.G., et al., Association between intraoperative blood transfusion and mortality and morbidity in patients undergoing noncardiac surgery.

Anesthesiology, 2011. 114(2): 283-92. 
10. Murad, M.H., et al., The effect of plasma transfusion on morbidity and mortality: a systematic review and meta-analysis. Transfusion, 2010. 50(6): 1370-83.

11. Fukamachi, A., H. Koizumi, and H. Nukui, Postoperative intracerebral hemorrhages: a survey of computed tomographic findings after 1074 intracranial operations. Surg Neurol, 1985. 23(6): p. 575-80.

12. Kalfas, I.H. and J.R. Little, Postoperative hemorrhage: a survey of 4992 intracranial procedures. Neurosurgery, 1988. 23(3): 343-7.

13. Touho, H., et al., Relationship between abnormalities of coagulation and fibrinolysis and postoperative intracranial hemorrhage in head injury. Neurosurgery, 1986. 19(4): 523-31.

14. Palmer, J.D., O.C. Sparrow, and F. lannotti, Postoperative hematoma: a 5year survey and identification of avoidable risk factors. Neurosurgery, 1994. 35(6): $1061-4$.

15. George, J.N. and S.J. Shattil, The clinical importance of acquired abnormalities of platelet function. N Engl J Med, 1991. 324(1): 27-39.

16. Pfanner, G., et al., [Preoperative evaluation of the bleeding history. Recommendations of the working group on perioperative coagulation of the Austrian Society for Anaesthesia, Resuscitation and Intensive Care]. Anaesthesist, 2007. 56(6): 604-11.

17. Werner, E.J., et al., Prevalence of von Willebrand disease in children: a multiethnic study. J Pediatr, 1993. 123(6): 893-8.

18. Fortuna, G.R., et al., The impact of preinjury antiplatelet and anticoagulant pharmacotherapy on outcomes in elderly patients with hemorrhagic brain injury. Surgery, 2008. 144(4): 598-603. 
19. Franko, J., et al., Advanced age and preinjury warfarin anticoagulation increase the risk of mortality after head trauma. J Trauma, 2006. 61(1): 07-10.

20. Gerlach, R., et al., Post-operative hematoma after surgery for intracranial meningiomas: causes, avoidable risk factors and clinical outcome. Neurol Res, 2004. 26(1): 61-6.

21. Rojkjaer, L.P. and R. Rojkjaer, Clot stabilization for the prevention of bleeding. Hematol Oncol Clin North Am, 2007. 21(1): 25-32.

22. Standeven, K.F., et al., Functional analysis of the fibrinogen Aalpha Thr312Ala polymorphism: effects on fibrin structure and function. Circulation, 2003. 107(18): 2326-30.

23. R., S., Pathophysiologie, Diagnostik und klinische Relevanz des Faktor-XIIIMangels. Egbring R, Seitz R, Wozniak G, Hrsg. Klinische Aspekte des FaktorXIII-Mangels, 1999. Basel: Karger: 7-15.

24. Ishida, F., et al., Spontaneous regression of the inhibitor against the coagulation factor XIII A subunit in acquired factor XIII deficiency. Thromb Haemost, 2010. 104(6): 1284-5.

25. Adam, E.H., et al., [Perioperative point-of-care coagulation testing--recently published studies]. Anasthesiol Intensivmed Notfallmed Schmerzther, 2013. 48(5): 346-50.

26. Ganter, M.T. and C.K. Hofer, Coagulation monitoring: current techniques and clinical use of viscoelastic point-of-care coagulation devices. Anesth Analg, 2008. 106(5): 1366-75.

27. Koscielny, J., et al., A practical concept for preoperative identification of patients with impaired primary hemostasis. Clin Appl Thromb Hemost, 2004. 10(3): 195-204. 
28. Jambor, C., et al., Multiple electrode whole blood aggregometry, PFA-100, and in vivo bleeding time for the point-of-care assessment of aspirin-induced platelet dysfunction in the preoperative setting. Anesth Analg, 2011. 113(1): 31-9.

29. Weber, C.F., et al., [Basic algorithm for Point-of-Care based hemotherapy: perioperative treatment of coagulopathic patients]. Anaesthesist, 2013. 62(6): 464-72.

30. Hartert, H., [Thrombelastography, a method for physical analysis of blood coagulation]. Z Gesamte Exp Med, 1951. 117(2): 189-203.

31. Calatzis, A., M. Heesen, and M. Spannagl, [Point-of-care testing of hemostatic alterations in anaesthesia and intensive care]. Anaesthesist, 2003. 52(3): 22937.

32. Haas, T., et al., Comparison of thromboelastometry $(\operatorname{ROTEM}(\mathrm{R}))$ with standard plasmatic coagulation testing in paediatric surgery. $\mathrm{Br} \mathrm{J}$ Anaesth, 2012. 108(1): 36-41.

33. Weber, C.F., et al., [Thrombelastometric detection of factor XIII deficiency]. Anaesthesist, 2008. 57(5): 487-90.

34. Schramko, A.A., et al., Role of fibrinogen-, factor VIII- and XIII-mediated clot propagation in gelatin haemodilution. Acta Anaesthesiol Scand, 2009. 53(6): 731-5.

35. Theusinger, O.M., et al., In vitro factor XIII supplementation increases clot firmness in Rotation Thromboelastometry (ROTEM). Thromb Haemost, 2010. 104(2): 385-91.

36. Hartert, H., Klin Wochenschr, 1948. 26(37-38): 577-83. 
37. Lang, T. and M. von Depka, [Possibilities and limitations of thrombelastometry/-graphy]. Hamostaseologie, 2006. 26(3 Suppl 1): 20-9.

38. ROTEM, 2011. TEM International GmbH, München, Deutschland: ROTEM: http://www.rotem.de/site/index.php?option=com_content\&view=article\&id=7\&lt emid=18\&lang=de,

39. Weber, C.F., et al., [Role of thrombelastometry for the monitoring of factor XIII. A prospective observational study in neurosurgical patients]. Hamostaseologie, 2011. 31(2): 111-7.

40. Lassen, B., et al., Surgical mortality at 30 days and complications leading to recraniotomy in 2630 consecutive craniotomies for intracranial tumors. Neurosurgery, 2011. 68(5): 1259-68.

41. Barker, F.G., 2nd, Craniotomy for the resection of metastatic brain tumors in the U.S., 1988-2000: decreasing mortality and the effect of provider caseload. Cancer, 2004. 100(5): 999-1007.

42. Sawaya, R., et al., Neurosurgical outcomes in a modern series of 400 craniotomies for treatment of parenchymal tumors. Neurosurgery, 1998. 42(5): 1044-55.

43. Biswas, A., et al., Coagulation factor XIII deficiency. Diagnosis, prevalence and management of inherited and acquired forms. Hamostaseologie, 2014. 34(2).

44. Dufner, G.S. and G.A. Marbet, [Factor XIII in man: a review]. Hamostaseologie, 2002. 22(1): 11-9.

45. Maak, B., et al., FXIII deficiency due to base exchange Thr 449 (ACT) --> Ile (ATT) in exon 11 of the factor $13 \mathrm{~A}$ gene. A cause of bleeding? Hamostaseologie, 2010. 30(3): 162-4. 
46. Korte, W., F. XIII in perioperative coagulation management. Best Pract Res Clin Anaesthesiol, 2010. 24(1): 85-93.

47. Seitz, R., et al., ETRO Working Party on Factor XIII questionnaire on congenital factor XIII deficiency in Europe: status and perspectives. Study Group. Semin Thromb Hemost, 1996. 22(5): 415-8.

48. Vrettou, C.S., et al., Factor XIII deficiency as a potential cause of supratentorial haemorrhage after posterior fossa surgery. Acta Neurochir (Wien), 2010. 152(3): p. 529-32.

49. Ternstrom, L., et al., Plasma activity of individual coagulation factors, hemodilution and blood loss after cardiac surgery: a prospective observational study. Thromb Res, 2010. 126(2): 128-33.

50. Jambor, C., et al., In vitro inhibition of factor XIII retards clot formation, reduces clot firmness, and increases fibrinolytic effects in whole blood. Anesth Analg, 2009. 109(4): 1023-8.

51. Nielsen, V.G., W.Q. Gurley, Jr., and T.M. Burch, The impact of factor XIII on coagulation kinetics and clot strength determined by thrombelastography. Anesth Analg, 2004. 99(1): 120-3.

52. Gorlinger, K., [Coagulation management during liver transplantation]. Hamostaseologie, 2006. 26(3 Suppl 1): S64-76.

53. Nielsen, V.G., et al., Thrombelastographic method to quantify the contribution of factor XIII to coagulation kinetics. Blood Coagul Fibrinolysis, 2007. 18(2): 145-50.

54. Dick, A., M. Schwaiger, and C. Jambor, [Thromboelastography/-metry and external quality control. Results of a pilot study]. Hamostaseologie, 2010. $30(2): 91-5$. 
55. Romlin, B.S., et al., Earlier detection of coagulopathy with thromboelastometry during pediatric cardiac surgery: a prospective observational study. Paediatr Anaesth, 2013. 23(3): 222-7.

56. Haas, T., et al., Reproducibility of thrombelastometry $(\operatorname{ROTEM}(\mathrm{R}))$ : point-ofcare versus hospital laboratory performance. Scand J Clin Lab Invest, 2012. 72(4): 313-7.

57. Weber, C.F., et al., Point-of-care testing: a prospective, randomized clinical trial of efficacy in coagulopathic cardiac surgery patients. Anesthesiology, 2012. 117(3): 531-47.

58. Toulon, P., et al., Point-of-care versus central laboratory coagulation testing during haemorrhagic surgery. A multicenter study. Thromb Haemost, 2009. 101(2): 394-401.

59. Venema, L.F., et al., An assessment of clinical interchangeability of TEG and RoTEM thromboelastographic variables in cardiac surgical patients. Anesth Analg, 2010. 111(2): 339-44.

60. Spalding, G.J., et al., [Bedside thrombelastography. Cost reduction in cardiac surgery]. Anaesthesist, 2007. 56(8): 765-71.

61. Mittermayr, M., et al., Hemostatic changes after crystalloid or colloid fluid administration during major orthopedic surgery: the role of fibrinogen administration. Anesth Analg, 2007. 105(4): 905-17.

62. Huraux, C., et al., Hemostatic changes in patients receiving hydroxyethyl starch: the influence of ABO blood group. Anesth Analg, 2001. 92(6): 1396401.

63. De Lorenzo, C., et al., Fibrinogen concentrate reverses dilutional coagulopathy induced in vitro by saline but not by hydroxyethyl starch $6 \%$. Anesth Analg, 2006. 102(4): 1194-200. 
64. de Jonge, E. and M. Levi, Effects of different plasma substitutes on blood coagulation: a comparative review. Crit Care Med, 2001. 29(6): 1261-7.

65. Chen, G., et al., Effects of two different hydroxyethyl starch solutions (HES200/0.5 vs. HES130/0.4) on the expression of platelet membrane glycoprotein. Acta Anaesthesiol Scand, 2006. 50(9): 1089-94.

66. Kind, S.L., et al., Is dilutional coagulopathy induced by different colloids reversible by replacement of fibrinogen and factor XIII concentrates? Anesth Analg, 2013. 117(5): 1063-71.

67. Schramko, A., et al., Hydroxyethylstarch and gelatin solutions impair blood coagulation after cardiac surgery: a prospective randomized trial. $\mathrm{Br} \mathrm{J}$ Anaesth, 2010. 104(6): 691-7.

68. Nielsen, V.G., Hydroxyethyl starch enhances fibrinolysis in human plasma by diminishing alpha2-antiplasmin-plasmin interactions. Blood Coagul Fibrinolysis, 2007. 18(7): 647-56.

69. Dekker, S.E., et al., Lysis onset time as diagnostic rotational thromboelastometry parameter for fast detection of hyperfibrinolysis. Anesthesiology, 2014. 121(1): 89-97.

70. Jámbor, C. and K. Görlinger, Einsatz von Antifibrinolytika bei Massivtransfusionen. Anästh Intensivmed, 2007. 48: 167-173. 


\section{Anhang}

\section{Patienteninformation}

Koagulopathie bei neurochirurgischen Eingriffen: Einfluss von kolloidaler versus kristalloider Infusionslösung 
Sehr geehrte Patientin, sehr geehrter Patient!

\section{Einleitung}

Ihnen steht ein operativer Eingriff am Gehirn bevor. Über die Risiken der Operation und der notwendigen Narkose wurden Sie bereits von einem Neurochirurgen und einem Anästhesisten aufgeklärt. Während der Operation ist es notwendig, sie kontinuierlich mit Flüssigkeit zu versorgen. Deswegen wird der Anästhesist Ihnen noch vor Beginn der Narkose und anschließender Operation eine Venenverweilkanüle legen und darüber kontinuierlich Flüssigkeit als Infusion geben. Dies hat zum einen den Sinn, Ihr Flüssigkeitsdefizit (Sie werden zu Beginn der Narkose schon mehrere Stunden nüchtern sein) auszugleichen, gewährleistet aber zum anderen auch, während der Operation entstehende Flüssigkeitsdefizite (z.B. durch Blutverlust) zu ersetzen.

Die Infusionslösungen sind industriell hergestellt und unterscheiden sich in Ihrer Zusammensetzung. Grundsätzlich werden sogenannte „kristalloide“ von „kolloidalen“ Infusionslösungen unterschieden. Beide Infusionslösungen können unter Einschränkungen einzeln als auch miteinander kombiniert infundiert werden.

Es ist bekannt, dass die Zufuhr sowohl von kristalloiden, als auch kolloidalen Infusionslösungen einen Einfluss auf die Blutgerinnung hat; hauptverantwortlich hierfür ist die Verdünnung des Blutes durch die Infusion, die zu einer Verringerung der Konzentration der Blutgerinnungsfaktoren und der für die Blutgerinnung wichtigen Blutplättchen führt. 
Worum geht es in der Untersuchung und was kommt auf Sie zu?

In der geplanten Untersuchung wollen wir den Einfluss von zwei Infusionsstrategien auf die Konzentration zweier ausgewählter Blutgerinnungsfaktoren und die Blutgerinnselfestigkeit untersuchen. Hierfür werden wir Ihnen an vier Meßzeitpunkten (vor, während (2x) und nach der Operation) Blut entnehmen (viermal $15 \mathrm{ml}$ ). Eine Analyse des Blutes soll Ergebnisse darüber liefern, ob durch die verschiedenen Infusionen die Gerinnungsfähigkeit des Blutes unterschiedlich beeinflusst wird. Zu diesem Zweck wollen wir die Konzentration zweier wichtiger Blutgerinnungsfaktoren (Fibrinogen und Faktor XIII) bestimmen und uns mit einer weiteren Labormethode (ROTEM) ein Bild von der Gerinnselfestigkeit machen.

Unsere Bitte an Sie ist, uns Ihre Einwilligung dafür zu geben, dass wir Ihre Daten, die wir bei diesen Untersuchungen gewinnen, anonymisiert und systematisch erfassen und auswerten und ggf. für Publikationen verwenden dürfen.

\section{Welche Risiken und Nebenwirkungen können auftreten?}

Die Risiken und Komplikationen, die sowohl durch die Anästhesie, als auch durch den chirurgischen Eingriff bestehen, haben Ihnen die behandelnden Ärzte der Chirurgie und Anästhesiologie bereits erläutert. Risiken und Nebenwirkungen, die Innen durch die Teilnahme an der Studie entstehen können, stehen im Zusammenhang mit den Blutentnahmen: Infektion der Einstichstelle, Blutung und Nervenläsionen.

\section{Vorzeitige Beendigung der klinischen Prüfung}

Ihre Teilnahme an der Untersuchung ist freiwillig, Sie können jederzeit, auch ohne Angabe von Gründen, Ihre Bereitschaft widerrufen, ohne dass Ihnen dadurch irgendwelche Nachteile für Ihre ärztliche Versorgung entstehen.

Wir hoffen, dass wir Ihnen mit Hilfe der Aufklärungsunterlagen und dem Gespräch mit dem Narkosearzt alle Fragen beantworten konnten.

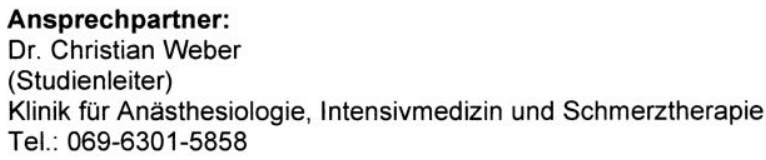

Abb. 13: Patienteninformation 


\section{Koagulopathie bei neurochirurgischen Eingriffen: Einfluss von kolloidaler versus kristalloider Infusionslösung}

Mir ist bekannt, dass bei dieser klinischen Prüfung personenbezogene Daten, insbesondere medizinische Befunde, über mich erhoben, gespeichert und ausgewertet werden sollen. Die Verwendung der Angaben über meine Gesundheit erfolgt nach gesetzlichen Bestimmungen und setzt vor der Teilnahme an der klinischen Prüfung folgende freiwillig abgegebene Einwilligungserklärung voraus, d. h. ohne die nachfolgende Einwilligung kann ich nicht an der klinischen Prüfung teilnehmen.

\section{Einwilligungserklärung zum Datenschutz (Arzneimittelgesetz)}

1) Ich erkläre mich damit einverstanden, dass im Rahmen dieser klinischen Prüfung erhobene Daten, insbesondere Angaben über meine Gesundheit, in Papierform und auf elektronischen Datenträgern in der Klinik für Anästhesiologie, Intensivmedizin und Schmerztherapie der Johann Wolfgang Goethe Universität Frankfurt am Main aufgezeichnet werden.

2) Außerdem erkläre ich mich damit einverstanden, dass die zuständigen inländischen und ausländischen Überwachungsbehörden in meine beim Prüfarzt vorhandenen personenbezogenen Daten, insbesondere meine Gesundheitsdaten, Einsicht nehmen, soweit dies für die Überprüfung der ordnungsgemäßen Durchführung der Studie notwendig ist. Für diese Maßnahme entbinde ich den Prüfarzt von der ärztlichen Schweigepflicht.

3) Die Einwilligung zur Erhebung und Verarbeitung meiner personenbezogenen Daten, insbesondere der Angaben über meine Gesundheit, ist unwiderruflich. Ich bin bereits darüber aufgeklärt worden, dass ich jederzeit die Teilnahme an der klinischen Prüfung beenden kann. Im Fall eines solchen Widerrufs meiner Einwilligung, an der Studie teilzunehmen, erkläre ich mich damit einverstanden, dass die bis zu diesem Zeitpunkt gespeicherten Daten ohne Namensnennung weiterhin verwendet werden dürfen, soweit dies erforderlich ist, um

a) Wirkungen des zu prüfenden Arzneimittels festzustellen,

b) sicherzustellen, dass meine schutzwürdigen Interessen nicht beeinträchtigt werden,

c) der Pflicht zur Vorlage vollständiger Zulassungsunterlagen zu genügen.

4) Ich erkläre mich damit einverstanden, dass meine Daten nach Beendigung oder Abbruch der Prüfung mindestens zehn Jahre aufbewahrt werden, wie es die Vorschriften über die klinische Prüfung von Arzneimitteln bestimmen. Danach werden meine personenbezogenen Daten gelöscht, soweit nicht gesetzliche, satzungsmäßige oder vertragliche Aufbewahrungsfristen entgegenstehen.

5) Ich bin über folgende gesetzliche Regelung informiert: Falls ich meine Einwilligung, an der Studie teilzunehmen, widerrufe, müssen alle Stellen, die meine personenbezogenen Daten, insbesondere Gesundheitsdaten gespeichert haben, unverzüglich prüfen, inwieweit die gespeicherten Daten für die in $\mathrm{Nr} .3$ a) bis c) genannten Zwecke noch erforderlich sind. Nicht mehr benötigte Daten sind unverzüglich zu löschen.

6) Ich bin mit der Aufzeichnung der im Rahmen der Studie an mir erhobenen Daten und ihrer anonymisierten Verwendung, $\mathbf{z}$. B. für Veröffentlichungen einverstanden.

Abb. 14: Datenschutzerklärung 


\title{
Einwilligungserklärung
}

zur Teilnahme an einer Untersuchung mit dem Thema

\section{Koagulopathie bei neurochirurgischen Eingriffen: Einfluss von kolloidaler versus kristalloider Infusionslösung}

\begin{abstract}
Name:
Vorname:

Pat. $\mathrm{Nr}$ :

Geburtsdatum:

Größe: Gewicht:

1.) Ich habe die von Frau/Herrn Dr. durchgeführte Aufklärung über Art und Durchführung sowie die möglichen Risiken der Untersuchung verstanden und hatte Möglichkeit, Fragen zu stellen. Ferner hatte ich Gelegenheit, die „Patienteninformation“ durchzulesen und Fragen dazu zu stellen. Die von mir gestellten Fragen wurden beantwortet. Ich konnte mir genügend Zeit nehmen, bevor ich meine Entscheidung zur Teilnahme an dieser klinischen Untersuchung getroffen habe.
\end{abstract}

2.) Die Teilnahme an dieser klinischen Untersuchung ist freiwillig. Ich kann jederzeit ohne Angabe von Gründen und ohne Nachteile meine Einwilligung zurückziehen. Eine derartige Entscheidung hat keinen Einfluss auf die weitere medizinische Behandlung.

3.) Eine Kopie dieser Einwilligungserklärung habe ich vom dem die Untersuchung durchführenden Arzt erhalten. Eine schriftliche Patienteninformation wurde mir ausgehändigt.

Ich erkläre mich bereit, an der Studie teilzunehmen

Eine Kopie der Patienteninformation, der Datenschutzerklärung und der Einwilligungserklärung habe ich erhalten. Das Original verbleibt beim Prüfarzt.

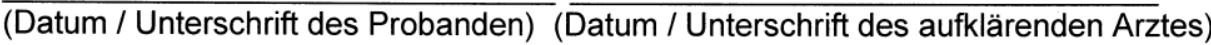

Abb. 15: Einwilligungserklärung 


\section{Schriftliche Erklärung}

Ich erkläre ehrenwörtlich, dass ich die dem Fachbereich Medizin der Johann Wolfgang Goethe-Universität Frankfurt am Main zur Promotionsprüfung eingereichte Dissertation mit dem Titel

„Stellenwert der Thrombelastometrie für das Monitoring von Faktor XIII“

in der Klinik für Anästhesiologie, Intensivmedizin und Schmerztherapie, Universitätsklinikum, Frankfurt am Main unter Betreuung und Anleitung PD Dr. med. Christian Weber ohne sonstige Hilfe selbst durchgeführt und bei der Abfassung der Arbeit keine anderen als die in der Dissertation angeführten Hilfsmittel benutzt habe. Darüber hinaus versichere ich, nicht die Hilfe einer kommerziellen Promotionsvermittlung in Anspruch genommen zu haben.

Ich habe bisher an keiner in- oder ausländischen Universität ein Gesuch um Zulassung der Promotion eingereicht. Die vorliegende Arbeit wurde bisher nicht als Dissertation eingereicht.

Vorliegende Ergebnisse der Arbeit wurden in folgendem Publikationsorgan veröffentlicht: PubMed, DIVI Abstractband

Weber CF, Sanders JO, Gerlach R, Platz J, Miesbach W, Hofstetter C

Untersuchung des Stellenwertes der Thrombelastometrie zur Detektion des perioperativ erworbenen Faktor XIII - Defizites bei neurochirurgischen Patienten DIVI Abstractband (ISBN: 978-3-00-032866-4; 2010; S. 104)

Weber CF, Sanders JO, Friedrich K, Gerlach R, Platz J, Miesbach W, Hanke AA, Hofstetter C

Role of thrombelastometry for the monitoring of factor XIII. A prospective observational study in neurosurgical patients.

Hamostaseologie, 2011. 31(2): p. 111-7. 


\section{Danksagung}

Diese Dissertation widme ich meinen Eltern Frau Antje Heinemann-Sanders und Herrn Jan-Dirk Sanders sowie meiner Schwester Friederike Sanders. Vielen Dank für die familiäre Unterstützung, ohne diese wäre mir das Medizinstudium nicht möglich gewesen.

Auch meiner Freundin Frau Annika Schreiber möchte ich für Ihre Unterstützung und Ihr Verständnis für die zeitweisen langen Abende am Schreibtisch danken.

Besonderer Dank gilt meinem Doktorvater und Betreuer PD Dr. med. Christan Weber, Oberarzt der Klinik für Anästhesiologie, Intensivmedizin und Schmerztherapie des Universitätsklinikums Frankfurt, Frankfurt am Main für die umfassende und engagierte Unterstützung.

Außerdem möchte ich mich bei dem Direktor der Klinik für Anästhesiologie, Intensivmedizin und Schmerztherapie des Universitätsklinikums Frankfurt, Frankfurt am Main, Professor Dr. med. Dr. phil. K. Zacharowski sowie dem Direktor der Klinik und Poliklinik für Neurochirurgie, Prof. Dr. med. V. Seifert für Ihre Unterstützung bedanken.

Offenbach, den 05.02.2015

Jan-Ove Sanders 